Check for updates

Cite this: Mater. Adv., 2020 1,2614

Received 26th June 2020

Accepted 24th August 2020

DOI: $10.1039 / \mathrm{d} 0 \mathrm{ma} 00455 \mathrm{c}$

rsc.li/materials-advances

\title{
Nanoparticle mediated alteration of EMT dynamics: an approach to modulate cancer therapeutics
}

\author{
Plaboni Sen, ${ }^{a}$ Muktashree Saha ${ }^{a}$ and Siddhartha Sankar Ghosh (iD *ab
}

\begin{abstract}
Metastasis is the cause of approximately $90 \%$ of cancer-related morbidities and mortalities, which is ascribed to the phenomenon of EMT (epithelial to mesenchymal transition). The pathological activation of EMT during tumour development results in the augmentation of metastasis and invasiveness of the primary tumours. Following EMT, the cells lose their epithelial characteristics and acquire a mesenchymal phenotype. This process involves the disruption of cell-cell and cell-matrix adhesion, changes in cellular polarity and remodelling of cytoskeleton, which are attributed to the enhanced pathogenesis in the tumours. EMT elicits various phenomena such as immune modulation, tumour immune escape, drug resistance and chemoresistance and is also responsible for poor prognosis in cancer. The use of nanoparticles as drug delivery vehicles or as cancer-inhibiting agents has been a topic of extensive research in today's world. Nanoparticles are used as efficient drug-delivering agents owing to their extremely small size and numerous merits such as biocompatibility, biodegradability, non-toxicity, ease of functionalization and encapsulation and enhanced permeability and retention (EPR) effect. Besides, nanoparticles can be modulated to be tumour specific by both passive and active targeting strategies and enhance the intracellular drug concentration in tumours while avoiding toxicity in healthy cells. Thus, the establishment and formulation of a novel nanomedicine might have remarkable and valuable contribution through specific or selective delivery of chemotherapeutic agents and/or inhibitors against EMT related pathways. Additionally, the utilization of their intrinsic cytotoxic activity or their use in the context of precision medicine can be allowed to modulate EMT by targeting the tumour microenvironment. This review deals with the various types of nanomaterials used to inhibit the phenomenon of EMT for achieving a successful anti-metastatic therapeutic regime.
\end{abstract}

\section{Introduction}

EMT is a conserved and dynamic multistep process implicated under a plethora of pathophysiological conditions including, embryonic development, wound healing and cancer. In malignancies, it not only imparts cancer cells the unique characteristics of migration and invasion but also results in the generation of cancer stem cells (CSCs) thereby contributing to chemoresistance and immune modulation along with a poor therapeutic outcome. The effect of EMT on cancer varies with respect to different tumours and their microenvironmental conditions. The EMT enables the cancer cells to migrate to a distant site through the bloodstream and undergoes MET (mesenchymal to epithelial transition), therefore,

\footnotetext{
${ }^{a}$ Department of Biosciences and Bioengineering, Indian Institute of Technology Guwahati, Guwahati-39, Assam, India.E-mail:sghosh@iitg.ac.in; Fax: +0361-258-2249; Tel: +0361-258-2206

${ }^{b}$ Centre for Nanotechnology, Indian Institute of Technology Guwahati,

Guwahati-39, Assam, India
}

resulting in the establishment of metastatic colonization, ${ }^{1}$ depicted in Fig. 1.

The cells undergoing EMT lose their apical-basal polarity, cell-to-cell adhesion, cytoskeleton reorganisation, and acquire the migratory mesenchymal phenotype such as increased motility and dissemination of cancer cells, which eventually enter the bloodstream in the form of circulating tumour cells (CTC). ${ }^{2}$ The loss of epithelial proteins, primarily E-cadherin, marks the process of EMT induction. However, other epithelial markers, which are downregulated during EMT, are cytokeratin, occludins, $\gamma$-catenin and laminins. Simultaneously, there is also overexpression of the mesenchymal markers such as Vimentin, N-cadherin, and $\alpha$-SMA ( $\alpha$-smooth muscle actin). The extent of loss of the epithelial characteristics is related to the aggressiveness of the malignancy, therefore, leading to poor prognosis and high rate of morbidity.

In the metastatic malignancies, upon induction of EMT, there is an increased expression of the matrix metalloproteases (MMPs) such as membrane type 1 (MT1)-MMP, MMP2 or 

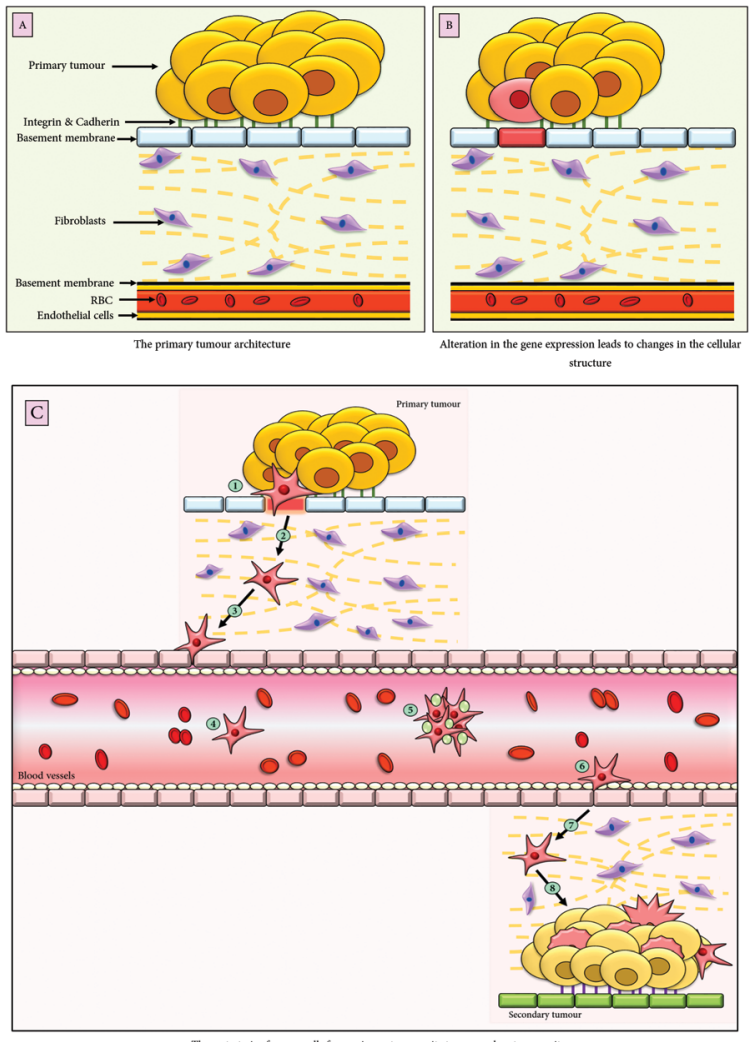

Fig. 1 Schematic representation of the epithelial to mesenchymal transition. (A) The tumour architecture; (B) upon external stimuli from the tumour niche, there is an alteration in the gene expression that results in the phenotypic change of the epithelial tumour cell; (C) following EMT, the epithelial cells acquire migratory mesenchymal characteristics; upon the secretion of matrix metalloproteases (MMPs) by the mesenchymal cells the basement membrane gets degraded (1), the mesenchymal cells then traverse the stromal cellular layers (2); the tumour cells possess PDGFR (platelet derived growth factor receptors), therefore, are chemo-attracted by the endothelial cells of the blood vessels secreting PDGF (3); following chemotaxis, the tumour cells intravasate into the blood vessels (4); (5) they travel to a distant site in the body either alone or in clusters, after being surrounded by the platelets which help them escape immune recognition, (6) the extravasation of the tumour cells through the blood vessels engenders secondary tumours (7), whereby, following the process of mesenchymal to epithelial transition (MET), the migratory cells acquire epithelial characteristics and give rise to an entirely new cluster of tumours (8).

MMP9 that facilitates the degradation of the basal lamina, therefore, enabling the cancer cells to migrate through the basement membrane. ${ }^{3}$ The cells undergoing EMT produce increased amounts of extracellular matrix proteins (collagen and fibronectin), which in association with the niche potentiates angiogenesis, invasion and metastasis, along with immune escape. ${ }^{4}$ EMT also allows the tumour cells to escape anoikis, i.e. death upon cell detachment; as a result, they flow through the lymphatic and blood vessels, thereby promoting metastasis. ${ }^{5}$

Specialized cell-cell junction proteins (claudin, occludin, cadherin, etc.), essential for maintaining the epithelial integrity, are lost upon the induction of the epithelial to mesenchymal transition. Besides, the cells undergoing EMT modulate their cytoskeletal organizations, enabling cell elongation and directional motility. ${ }^{6}$
EMT is attributed to the remodelling of actin filaments which are mediated by regulatory proteins, such as moesin, a crosslinker protein between the plasma membrane and actin-based cytoskeleton. ${ }^{7}$ It is followed by the formation of a protrusive pseudopodia structure such as invadopodia and lamellopodia (sheet like membrane protrusions) that have spike like projections at their edges known as filopodia. ${ }^{8}$ These actin-rich protrusions expressed on the cellular edges exert proteolytic functions and are customized for the degradation of the ECM. These actindependent pseudopodial protrusions in the tumour cell are critical elements of mesenchymal cell migration that eventually leads to cancer metastasis. ${ }^{8}$ The role of cytoskeletons in EMT was reviewed by Fefe $e t$ al. and Sun et al. ${ }^{9,10}$ The RHO/RACK pathway in synergism with the FAK pathway is known to regulate the actin/cytoskeleton dynamics during EMT. ${ }^{11}$ While RhoA promotes actin stress fibre formation, the RAC1/CDC42 fosters the genesis of lamellopodia and filopodia, (reviewed by Lamouille et al.).

Decades of ongoing research have provided us with established evidence regarding the role of EMT in metastasis and invasion of the cancer cells. However, the lack of a suitable delivering system targeting EMT remains a major concern. This review deals with the utilization of synthetic and natural nanomaterials which are either used as EMT inhibitors or as a drug delivery vehicle to inhibit the various EMT potentiating factors.

\section{The factors facilitating EMT}

The factors contributing to the loss of the epithelial phenotype are either related to zinc-finger binding transcription factors (Snai1/Snail, Snai2/Slug, ZEB1, and ZEB2) or the basic helixloop-helix factors (Twist1 and Twist2). Besides, other factors downregulating the epithelial characteristics include GSK-3 $\beta$, TCF/LEF, $\beta$-catenin, SUMO2, etc. EMT is also induced by humoral factors [such as epidermal growth factor $(E G F),{ }^{12}$ fibroblast growth factor $(F G F),{ }^{13}$ hepatocyte growth factor $(H G F)^{14}$ and platelet-derived growth factor $(P D G F)^{15}$ ] acting via receptor tyrosine kinases, ${ }^{16}$ secreted signalling molecules lying downstream of the $\mathrm{Wnt}^{17,18}$ and Notch $^{19}$ pathway, MAPK pathway ${ }^{20}$ and cytokines such as transforming growth factor- $\beta$ (TGF $\beta)^{21}$ that are abundantly expressed in the tumour microenvironment. Additionally, hypoxia and inflammation are also known to regulate EMT. ${ }^{22,23}$

The transcription factors (Snail, Slug, Twist, and ZEB) are known to downregulate the expression of E-cadherin along with numerous other epithelial markers and also aid in the acquisition of mesenchymal markers. Damian Medici et al. reported that among the three Snail family of proteins, Snai1 (known as Snail) and Snai2 (known as Slug) promote the expression of $\beta$-catenin and TCF4 transcription complexes that bind to the promoters of TGF $\beta 3$, thereby increasing its transcription. Furthermore, the TGF $\beta 3$ signalling increases the LEF1 (lymphoid enhancer factor) gene expression, causing the formation of $\beta$-catenin and LEF1 complexes that initiate EMT without upstream signalling pathways. ${ }^{24}$ The Snail family of proteins binds to the E-box of the E-cadherin promoter through their 
carboxyl-terminal zinc-finger domain and represses the expression of E-cadherin. Multiple signalling pathways activate Snail expressions such as Wnt, Notch, ${ }^{25}$ and TGF $\beta .^{26}$ The Snail proteins interact with transcriptional regulators such as ETS1 and SMAD3/4 which are activated by the MAPK/PI3K signalling pathways and results in the upregulation of MMP expression. They are also known to regulate the TGF $\beta$ mediated repression of E-cadherin and occludin, therefore promoting EMT. ${ }^{27,28}$

The basic helix-loop-helix transcription factors (Twist1 and Twist2) facilitate the downregulation of epithelial genes and activate the expression of mesenchymal genes. They are known to repress the E-cadherin expression and activate the N-cadherin expression, in alliance with other regulatory proteins. One such association is of Twist with Bmi1 that results in the recruitment of the PRC2 gene, which in turn represses the gene promoters of E-cadherin and p16. ${ }^{29}$ Under hypoxic conditions, HIF1 $\alpha$ in association with $\beta$-catenin induces the expression of the Twist1 gene. In association with Snail, Twist gets phosphorylated by MAPK, which protects it from ubiquitin-mediated degradation, thereby increasing its activity. ${ }^{30}$

The zinc finger E-box binding homeobox-1 and -2 known as ZEB1 and ZEB2, respectively, bind to the regulatory gene sequences at the E-boxes of the gene promoters and can either repress or activate the transcription of those genes. They also bind to the transcriptional activators such as p300/CBP associated factors, which transcriptionally activates certain genes, including Smad and facilitates the TGF $\beta$ pathway. ${ }^{31}$ Their expression follows Snail activation. Besides, it is also induced upon the cooperation of Twist and Snail genes ${ }^{32}$ and also by TGF $\beta,{ }^{32}$ Wnt proteins and RAS/MAPK signalling pathways. ${ }^{33}$

Among the different signalling pathways, the developmental pathways such as the Notch, ${ }^{34}$ Wnt, hedgehog, ${ }^{35}$ MAPK, and $\mathrm{AKT}^{36}$ pathways are the most common inducers of EMT. However, among the various growth factors, TGF $\beta$ is the potent inducer of EMT, having established crosstalk with numerous other pathways downstream of it, in Smad and non-Smad dependent manner. ${ }^{37,38}$ These pathways are known to facilitate either the expression of the transcriptional factors (Snail, ZEB and Twist) or are associated with the expression of downstream genes (MMPs, tight junction proteins, etc.) that further regulates EMT progression. The pathways potentiating EMT are illustrated in Fig. 2; however, a detailed mechanism of the individual pathway in regulating EMT is beyond the scope of this review.

\section{Exosomes mediated EMT in cancer}

Exosomes are nano-sized extracellular vesicles secreted by a variety of cells, which increase in case of stress or diseased conditions like cancer. Initially, it was known to eliminate waste from the cells, but researchers have found that they also act as a mediator for various cell-to-cell communications. ${ }^{39}$

Exosomes carry biologically active molecules across the circulation and act as intracellular communicators that mediate orchestration of the local and distant tumour microenvironment. It results in the initiation of metastasis by facilitating EMT, demonstrated in Fig. 3. It is known to regulate various EMT-related pathways and also enhances the progression of metastasis and invasiveness of the cancer cells by exosome-mediated delivery of proteins and nucleic acids.

The tumour-derived exosomes (TDEs) carry pro-EMT cargoes which contain EMT inducer molecules (TGF $\beta$, TNF $\alpha$, IL-6, TSG101, AKT, ILK1, $\beta$-catenin, hepatoma-derived growth factor, casein kinase II (CK2), annexinA2, integrin 3, caveolin-1, matrix metalloproteases (MMPs) and hypoxia inducible factors) that facilitate EMT. ${ }^{40}$ Exosomes also contain Wnt proteins that result in the activation of the Wnt signalling pathway in recipient cells. The Wnt proteins are known to inhibit glycogen synthase kinase-3 $\beta$ (GSK3 $\beta$ ) that stabilizes $\beta$-catenin, which in turn promotes the gene expression program, favouring EMT. ${ }^{41}$

In some cases, it has been observed that highly metastatic hepatocellular carcinoma (MHCC97H) cell-derived exosomes are taken up by low metastatic hepatocellular carcinoma (HCC) cells, thus, resulting in the subsequent increase of invasiveness and malignant behaviour of the recipient cells. ${ }^{42}$ Exosomes derived from melanoma cells trigger the switch in phenotypes in primary melanocytes via the paracrine/autocrine mediated signalling, resulting in the activation of the MAPK pathway, thereby promoting metastasis.

Some of the miRNA modulators of EMT such as LET-7i, miR191 and let-7a, present in serum exosomes, are also involved in the process of switching from non-melanoma subjects to differentiating Stage-I melanoma patients, providing evidence against melanoma derived exosomes promoting EMT by modulating the tumour microenvironment. ${ }^{43}$ Exosomes carrying mi-499a-5p, derived from a highly metastatic cell line, enhanced cell proliferation, migration and EMT via the MTOR pathway in lung adenocarcinoma. ${ }^{44}$ Exosomes derived from breast cancer cell lines, containing miR-105, downregulated the expression of the ZO-1 protein, a tight junction protein in endothelial monolayer cells and resulted in the enhanced vascular permeability in lung and brain metastasis. ${ }^{45}$

It was also observed that TGF $\beta 1$ present in CAF (Cancer Associated Fibroblast) derived exosomes triggers the belligerent behaviour in ovarian cancer cells by activating the SMAD pathway, which is suggestive of the fact that targeting the CAF exosomes would result in the potential treatment of ovarian cancer. $^{46}$

\section{The EMT heterogeneity}

Metastasis occurs through repeated cycles of EMT and MET, as depicted in Fig. 4. The EMT occurs through distinct intermediate steps that evolve through the epithelial state to a hybrid E/M (hybrid epithelial/mesenchymal) state following a complete mesenchymal state. All the EMT cells possess similar tumour propagating capacity and display variations in their cellular plasticity, invasiveness and metastatic potential. These different states of EMT exhibit variation in their signalling pathways, transcriptional factors and their niches. ${ }^{47}$

Similarly, the mesenchymal to epithelial transition (MET) is the reverse process of EMT that involves the transition from the motile, multipolar, spindle-shaped mesenchymal cells to planar polarized epithelial cells. It also follows a series of distinct 


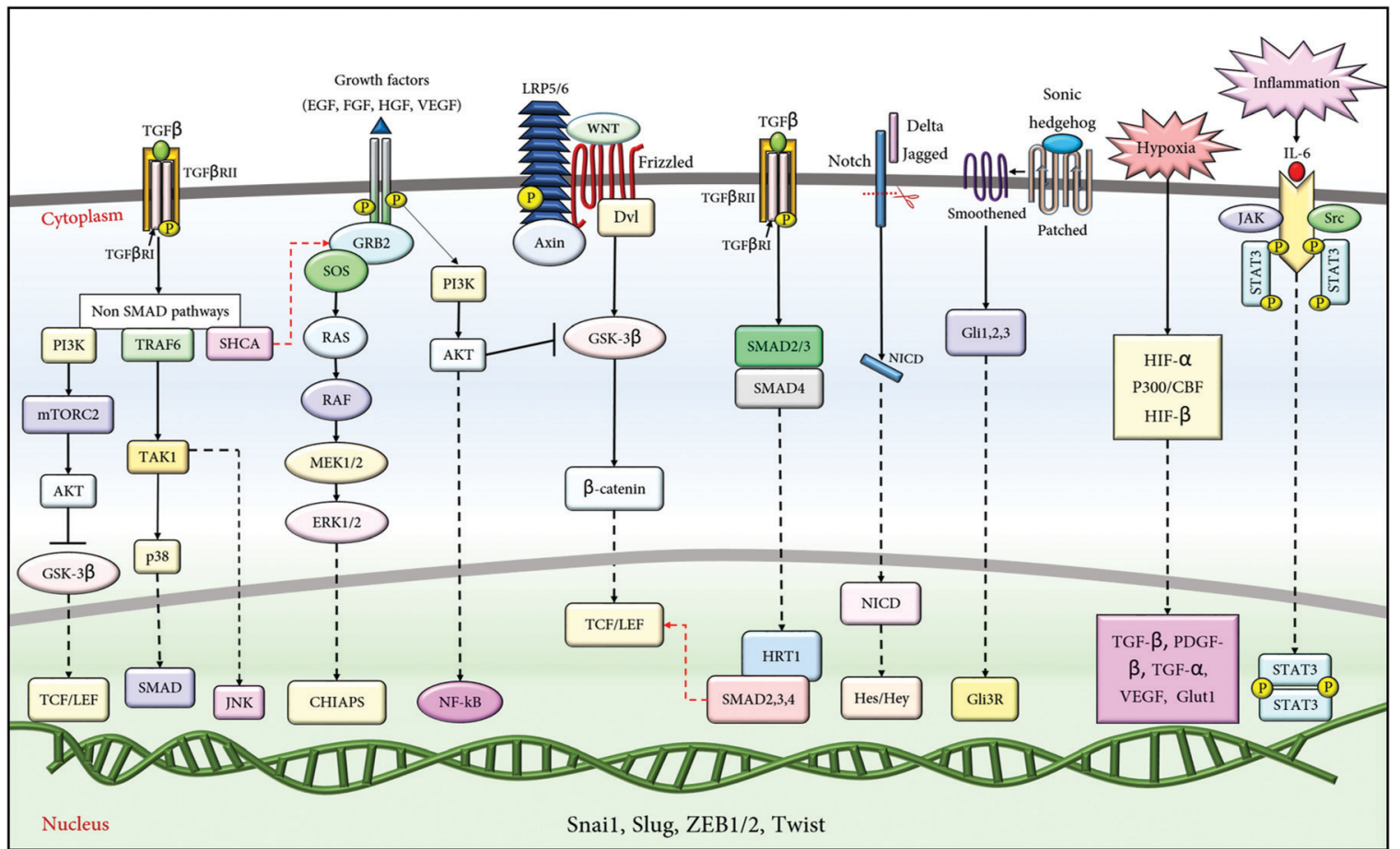

Fig. 2 The pathways facilitating EMT. The pathways known to induce EMT by activating the downstream pathway genes and transcription factors are the Sonic hedgehog pathway, Smad-dependant and independent TGF $\beta$ pathway, Receptor tyrosine kinase pathway, Notch pathway, and Wnt pathway. Additionally, hypoxia and inflammation, the factors of the tumour microenvironment, are also known to potentiate the epithelial to mesenchymal transition.

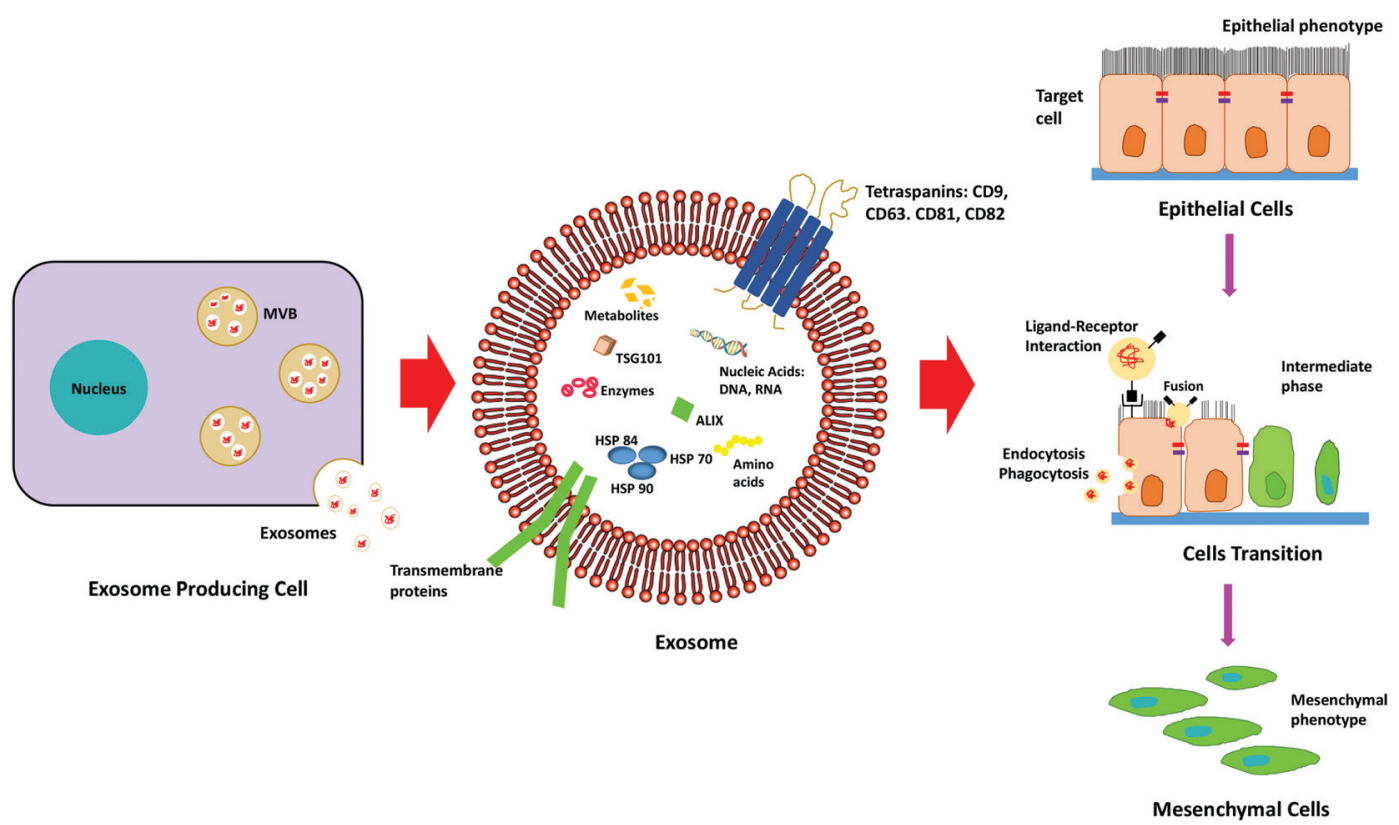

Fig. 3 Schematic representation depicting the role of exosomes in the progression of EMT.

transformations whereby, the mesenchymal cells traverses through the hybrid $\mathrm{M} / \mathrm{E}$ (hybrid mesenchymal/epithelial) state, following a complete epithelial state. Thus, researchers have syncretized the notion that cancer cells utilize the phenomenon of EMT for invasion and dissemination while MET helps in establishing the metastatic outgrowth. ${ }^{48}$
It has been observed that tumour cells exhibiting hybrid E/M phenotypes allow the migration of the adherent cells in the form of a cluster of circulating tumour cells (CTCs). It does so by exhibiting residual cell-cell interactions that enable collective migration of the cellular cluster. The key player responsible for the formation of these CTC clusters is the jagged mediated 


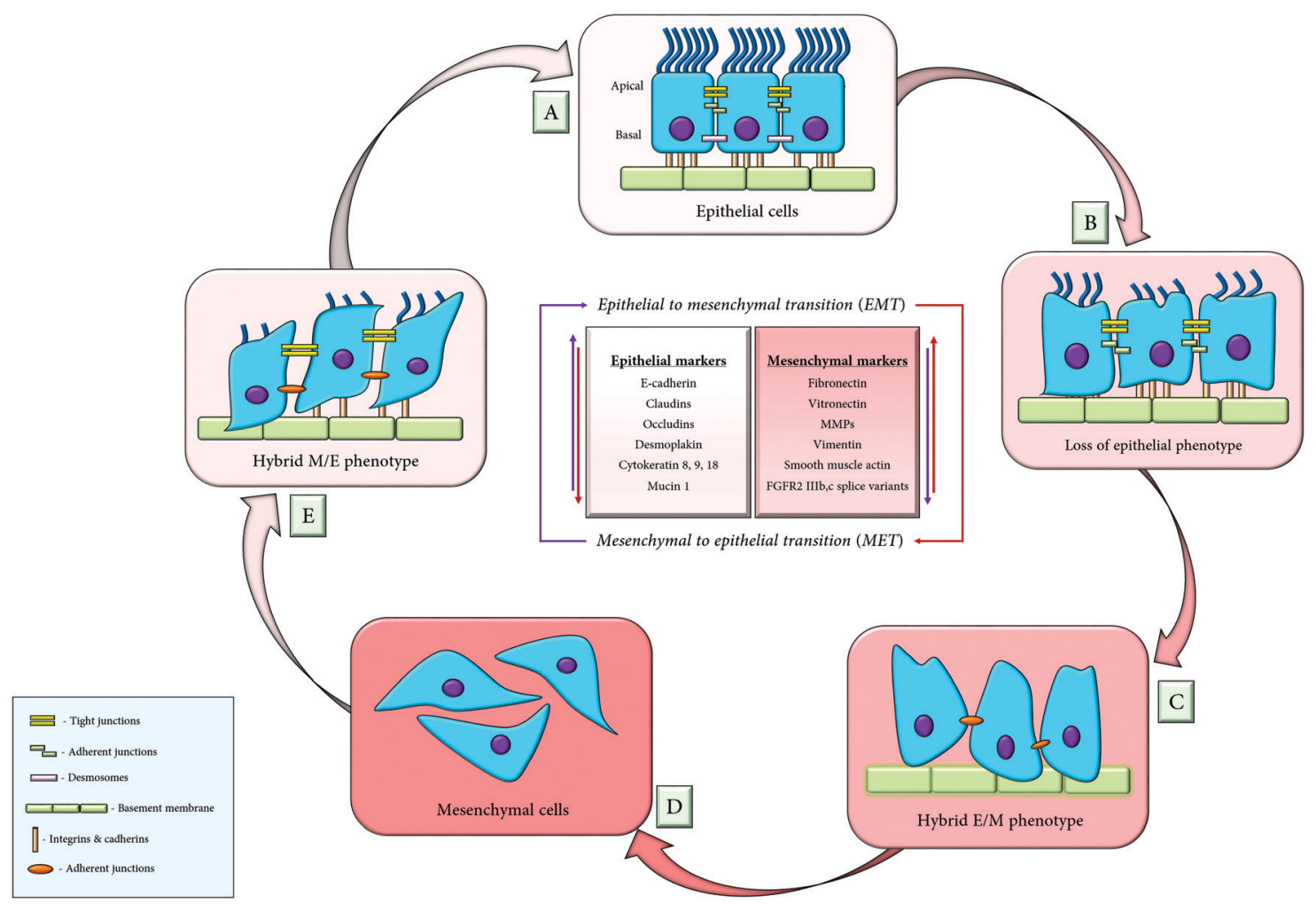

Fig. 4 The consortium of EMT and MET; (A) the epithelial cells are in close association with adjacent cells and the basement membrane, through their adherent junctions; (B) loss of epithelial markers results in the dissociation of the adherent junctions, which enables the epithelial cells to acquire a hybrid epithelial/mesenchymal (E/M) phenotype (C); (D) cells attain migratory mesenchymal phenotypes upon procuring mesenchymal markers, thus depicting the phenomenon of EMT. The mesenchymal to epithelial transition (MET) is the reverse process of EMT, whereby the cells lose their mesenchymal characteristics and initially acquires partial (hybrid M/E) epithelial phenotype (E), followed by a complete epithelial phenotype (F).

Notch signalling, ensuing lateral induction; the phenomenon whereby the adjacent cells acquire uniform phenotypes. As a result, they remain bound to each other in the form of clusters.

The cell fate determination among these three phenotypes is regulated by a circuit, composed of two mutually interconnected feedback loops that consist of two transcription factors (Snail and ZEB) and two micro-RNAs (miRNA-34 and miRNA-200): (i) the miRNA-34/Snail and (ii) the miRNA-200/ZEB. These two feedback loops are responsible for exhibiting any one of the three phenotypes of the EMT cells. High miRNA-200, high miRNA-34, low Snail and low ZEB exhibit the epithelial phenotype, and low miRNA200, low miRNA-34, high Snail and high ZEB mark the mesenchymal phenotype while low miRNA-34, low ZEB, high Snail and high miR200 evince the hybrid E/M state. ${ }^{49,50}$

Therefore, it has been imperative for us to identify these transition states and effectively eliminate the origin of these phenotypes, which might be a crucial step in abrogating various tumorigenic properties associated with a poor outcome in patients.

\section{What do nanoparticles deliver?}

Nanoparticles delivering nucleic acids to revert EMT: Therapeutic nucleic acids explored as anti-cancer agents hold promising results in the inhibition of EMT. However, the main problem concerning the nucleic acids is their intracellular stability and successful delivery into the tumour specific cells. The most commonly used nucleic acids for therapeutics are RNA based, which usually fall in either of the two categories: siRNA and miRNA that might be used against EMT related genes and transcription factors.

Nanoparticles delivering chemotherapeutic drugs in addition to EMT inhibiting agents: Chemotherapeutic drugs (doxorubicin, cisplatin, paclitaxel, daunorubicin, carboplatin, etc.) are delivered either by loading them into the nanoparticles following monotherapy or used in combination therapy with some other inhibitor loaded nanoparticles.

Nanoparticles delivering pathway inhibitors: There are numerous pathways that aid in the induction of EMT (Wnt, Notch, TGF $\beta$, MAPK, etc.). The use of pathway inhibitor loaded nanoparticles, either in monotherapy or in combination therapy, is highly efficient in curbing the effects of EMT, thus providing a dual therapeutic regime that results in the retraction of various hallmarks of malignancies.

\section{Nanoparticle mediated EMT inhibition}

Depending on the structural and physio-chemical properties, the nanomaterials possess unique abilities to either intrinsically inhibit EMT or transmit drugs encapsulated within itself to inhibit the EMT facilitating factors. 
Inorganic nanoparticles (metal and metal oxide nanoparticles), hybrid nanocomposites (Inorganics-In-Organics and Organics-In-Inorganics), carbon derived nanoparticles and polymeric nanoparticles (chitosan, micelle, PLGA) have exhibited remarkable capabilities of EMT inhibition. In addition to synthetic nanoparticles, natural nanomaterials (liposomes and exosomes) also possess exceptional characteristics as EMT inhibitors or as a drug delivery vehicle. Besides, there are numerous other nanoparticles that mediate the inhibition of EMT and metastasis, either encapsulated with a single inhibiting agent or following a co-therapeutic module (i.e., encapsulated with a combination of drugs or inhibitors), are discussed in Table 1.

\section{Inorganic metallic nanoparticles}

Gold nanoparticles (Au NPs) have attracted a wide variety of biomedical applications due to their remarkable properties such as ease of synthesis and characterisation, low cytotoxicity, biocompatibility and stability. They also possess exceptional properties of surface modifications pertaining to their strong ability to bind thiol (-SH-) and amino (-NH2-) group containing molecules, which in turn facilitates the binding of proteins through their cysteine and lysine residues. ${ }^{51}$

With respect to EMT, the unmodified gold nanoparticle has shown its ability to inhibit the proliferation, and migration by downregulating the mesenchymal markers (Snail, N-cadherin, Vimentin) and upregulating the epithelial markers (E-cadherin) in melanoma blood vessels, ${ }^{52}$ along with the reversal of epithelial plasticity by inhibiting MAPK pathways in ovarian cancer cells in vitro. It does so by reducing the secretion of several proteins involved in the induction of EMT. However, the mechanism is still not precise and needs to be examined further. Besides, the AuNPs have shown to normalize the tumour vasculature, increase blood perfusion, alleviate hypoxia and downregulate metastasis by inhibiting the expression of MMP2 and cMyc. ${ }^{52}$

They also increase the sensitivity of cancer cells towards chemotherapeutic drugs such as cisplatin, and gemcitabine, resulting in the suppression of cancer stem cell properties and inhibition of EMT in epithelial ovarian cancer cells and pancreatic cells in vitro, by downregulating $\mathrm{AKT} / \mathrm{NF}-\kappa^{53}$ and MAPK signalling, ${ }^{54}$ respectively. Simultaneously, photo-thermal therapy with gold nanorods has also shown to inhibit EMT and collective cell migration by altering the cell junction proteins and actin networks. ${ }^{55}$ A minimal dose of PEG-coated Au NPs sensitizes the solid tumours to cold plasma by inhibiting the PI3K/AKT signalling pathway and activating the p53 pathway, thus resulting in the inhibition of proliferation and EMT both in vivo and in vitro. ${ }^{56}$

Au NPs in conjugation with Quercetin inhibit angiogenesis, cell invasion and EMT via EGFR/VEGFR2 mediated pathways in breast cancer cells in vitro. ${ }^{57}$ In conjugation with dexamethasone (DSH), a synthetic glucocorticoid and an anticancer drug Withaferin A, the nanoconjugate sensitizes cancer cells to chemotherapeutics by downregulating the phosphoAKT signalling pathway, which in turn results in the downregulation of the ABCG2 drug transporters, induction of apoptosis in the glucocorticoid receptor dependant cancer cells and inhibiting proliferation of murine melanoma that ultimately reverses EMT. $^{58}$

\section{Other metallic nanoparticles}

Silver nanoparticles (Ag NPs) exhibit antimicrobial effects in addition to anti-tumour activity. They have exhibited their ability to inhibit the proliferation of breast cancer cell lines in vitro by inducing apoptosis. ${ }^{59}$ Gallic acid coated silver NPs inhibit EMT and sensitize the cancer cells to the radiation-induced metastasis; they were able to downregulate the EMT markers such as Vimentin, $\mathrm{N}$-cadherin, and Snail and upregulate the E-cadherin expression in non-small cell lung cancer (NSCLC). ${ }^{60}$ However, the use of Ag-NPs is limited due to their cytotoxicity upon long term exposure and their ability to induce proliferation and metastasis, as observed in colon adenocarcinoma cancer. ${ }^{61}$

Selenium nanoparticles (Se NPs) have anticancer and immunomodulatory properties and affect the redox machinery of cancer and healthy cells in a differential manner, (reviewed by Khurana et al.). ${ }^{62}$ It has been reported that curcumin loaded selenium NPs induce apoptosis, downregulate NF-kB signalling and inhibit EMT in Ehrlich's ascites carcinoma mouse model and colorectal carcinoma in vitro. ${ }^{63}$ In combination therapy with doxorubicin loaded nanoparticles and curcumin loaded Selenium nanoparticles, inhibition of EMT associated proteins (Snail, Vimentin, MMP2/9, and N-cadherin) was observed along with the increased ROS levels and induction of cell cycle arrest and apoptosis in colorectal cancer cells, in vitro. ${ }^{64}$

\section{Metallic oxide nanoparticles}

Metal oxide nanoparticles have also been exploited to deliver a variety of chemotherapeutic drugs and small molecule inhibitors as a therapeutic module.

Titanium dioxide nanoparticles $\left(\mathrm{TiO}_{2}\right.$-NPs) were found to suppress the EMT mediated cell migration and invasion, without exhibiting cytotoxicity. ${ }^{65} \mathrm{Li}$, Peng et al. observed that the $\mathrm{TiO}_{2}$-NPs and silicon dioxide nanoparticles ( $\mathrm{SiO}_{2}$-NPs) exhibit unique properties to block fibrosis in the hepatic cells. They also found that the $\mathrm{TiO}_{2}$ and $\mathrm{SiO}_{2}$ NPs inhibit the expression of collagen I and $\alpha$-Smooth muscle actin ( $\alpha$-SMA) by inducing lysosomal degradation of the TGF receptor complex (TGFRI and TGFRII). Additionally, the inhibition of the TGFR complex resulted in the downregulation of TGF-target genes, that further inhibited migration and invasion upon suppression of the epithelial to mesenchymal transition. ${ }^{65,66}$

Wahab et al. observed that zinc oxide nanostructures (ZnO-NS) downregulate various EMT markers and exert a cytotoxic effect against various cancer cells. This also led to the suppression of migration and invasion and induced apoptosis. Zinc nano-rods have shown to induce cancer cell death mediated by oxidative stress and caspase dependant pathways in glioblastoma, lung cancer and thyroid cancer. ${ }^{67}$

Wang and co-workers demonstrated that cuprous oxide $\left(\mathrm{Cu}_{2} \mathrm{O}-\mathrm{NP}\right)$ nanoparticles inhibited proliferation and metastasis of melanoma by targeting mitochondria, which resulted in the release of cytochrome $C$, which in turn induced apoptosis by activating Caspase 3 and caspase $9 .^{68}$

Iron oxide nanoparticles (IONPs) possess enhanced biocompatibility, biodegradability and properties like super paramagnetism. 
Table 1 Nanoparticles and their role in EMT inhibition

\begin{tabular}{ll}
\hline Sl & \\
no. & Nanoparticle \\
\hline $1 \quad \begin{array}{l}\text { Mesoporous silica (MSN); PEG-PLA } \\
\text { micelles (Mic) }\end{array}$
\end{tabular}

2 Layered double hydroxide nanocomposite

3 Zinc arsenite nanoparticles

$4 \quad$ Albumin based nanoparticles

5 Polyethyleneimine (PEI) coated meso- Conjugated with porous silica nanoparticles

6 Polyamidoamine dendrimers (PAMAM) conjugated mesoporous silica nanoparticles

7 Polyethyleneimine-coated mesoporous TWIST1 siRNA silica

8 Polyamidoamine dendrimers (PAMAM)

$9 \quad$ Lipid ECO based nanoparticles

10 Amino lipid ECO carrier (RGD-PEGECO/siDANCR) nanoparticles

11 Polyethylene glycolpolyethyleneimine-chlorin e6 (PEGPEI-Ce6) nanoparticles

12 Cationic solid lipid nanoparticles (SLN)

13 Gelatin nanoparticles

In combination therapy (EIN) Arsenic trioxide

Arsenic trioxide hyaluronic acid SiRNA

Loaded with $\beta 3$ peptides SiRNA
Effects

Cancer type

Ref.

Conjugated with Epigallocatechin gallate and iron nanocomplex

Suppression of metastasis and Mouse breast can- Fan et al. ${ }^{108}$ inhibition of EMT markers (MMP2/9, cer (4T1) cells; VEGF, Vimentin) and upregulation of female BALB/c mice

Loaded with etoposide Downregulation of EMT TFs (Snail, Glioblastoma stem Accepted N-cadherin) which inhibits metastasis; cells (GSC) downregulation of stem cell markers U87MG; female (Sox2, Nanog, Oct4, Nestin); inhibition BALB/c nude mice of PI3K/AKT/mTOR pathway and upre-

gulation of Wnt/GSK3 $\beta / \beta$-catenin

Encapsulated in the Suppression of tumour initiation and MHCC97L and silicon dioxide $\left(\mathrm{SiO}_{2}\right)$ proliferation; downregulation of EMT Hep3b, liver cancer matrix and loaded with markers (Vimentin, Slug) thereby sup- cell lines pressing metastasis; downregulation of SHP/JAK2/STAT3 signalling pathway Inhibition of cell invasion and EMT by Nasopharyngeal downregulation of $\mathrm{N}$-cadherin, Vimen- carcinoma tin and upregulation of E-cadherin Xenograft model Reduced tumorigenesis

Ovcar8 ovarian Loaded with Twist1 siRNA and Cisplatin Loaded with Twist1 siRNA and Cisplatin

Reduced metastasis and cancer cell line $\mathrm{NSG}^{a}$ mice model

chemoresistance

Inhibition of EMT followed by chemo- A2780R and Ovcar8 Roberts et al. ${ }^{113}$ sensitivity to Cisplatin Ovarian cancer cell lines; $\mathrm{NSG}^{a}$ Mice model

Reversal of EMT and inhibition of migration MDA-MB-435 Mela- Finlay et al. ${ }^{114}$ noma cells

Downregulation of Vimentin and CCL2 $\mathrm{NSG}^{a}$ mice model preventing tumour growth and metastasis

Loaded with Twist1

Reduction of cell migration and invasion upon reversal of EMT

cells;

$\mathrm{NSG}^{a}$ Mice model

Downregulation of PAL1 and integrin siRNA and modified with RGD

$\mathrm{N}$-cadherin, with upregulation of $\mathrm{MDA}$

Y. Lu et al. ${ }^{111}$

Shahin et al. ${ }^{112}$

-cadherin and CK19

Inhibition of TGF $\beta$-mediated invasion Female BALB/c

Encapsulated by PEG and functionalized with RGD peptides Loaded with long non- of $\beta$-catenin, ZEB1, STAT proteins, coding RNA, DANCR Survivin and followed by inhibition
Loaded with Wnt-1 SiRNA

Encapsulated with STAT3 decoy Oligodeoxynucleotides (ODN)

Conjugated with EGFR Downregulation of MMP2/9, targeting antibodies and AXL siRNA such as Wnt1, $\beta$-catenin

Inhibition of EMT and cell proliferation dynamic therapy (PDT) and Vimentin inhibition of EMT and EMT Inhibition of cell proliferation, migration and invasion, followed by inhibition of EMT; downregulation of the WNT pathway

Downregulation of Wnt pathway genes

Downregulation of Wnt pathway genes

Increased sensitivity towards photo-

Induction of apoptotic and autophagy mediated cell death; inhibition of

2780 and SKOV3 ovarian cancer cell

nude mice

MDA-MB-231 and Z. Lu et al. ${ }^{117}$ BT549

$\mathrm{TNBC}^{a}$ cell line; MDA-MB-231 and BT549 xenograft model

Cell carcinoma

TAT3 pathway; inhibition of invasion lines and EMT by downregulation of Snail

$\mathrm{N}$-cadherin, Vimentin; signifying the

Overcoming chemoresistance to

H820 and H1975

Suresh et al. ${ }^{120}$
Y. Ma et al. ${ }^{119}$ nude athymic mice; tyrosine kinase inhibitors; inhibition NSCLC $^{a}$ cell lines of the mTOR pathway and overexpression of p53

Erlotinib-resistant 
Table 1 (continued)

\begin{tabular}{|c|c|c|c|c|c|}
\hline $\begin{array}{l}\text { Sl } \\
\text { no. }\end{array}$ & Nanoparticle & $\begin{array}{l}\text { In combination } \\
\text { therapy }\end{array}$ & Effects & Cancer type & Ref. \\
\hline 14 & $\begin{array}{l}\text { Polyethylene glycol-polyethylenimine- } \\
\text { magnetic iron oxide NPs (PEG-PEI- } \\
\text { IONPs) }\end{array}$ & $\begin{array}{l}\text { Loaded with } \\
\text { microRNA-21 antisense } \\
\text { Oligonucleotides and } \\
\text { gemcitabine }\end{array}$ & $\begin{array}{l}\text { Upregulation of tumour suppressor } \\
\text { genes, PTEN and PDCD4; inhibition of } \\
\text { EMT, invasion, migration and cell } \\
\text { proliferation; induction of apoptosis }\end{array}$ & $\begin{array}{l}\text { PANC- } 1 \text { and MIA } \\
\text { PaCa-2 Pancreatic } \\
\text { cancer cells; female } \\
\text { BALB } / c \text { nude mice }\end{array}$ & Y. Li et al. ${ }^{121}$ \\
\hline 15 & $\begin{array}{l}\text { Lipid-polymer hybrid nanoparticles } \\
\text { modified with } \mathrm{CPP}^{a}\end{array}$ & Afatinib; miR-139 & $\begin{array}{l}\text { Inhibition of EMT; increased sensitivity } \\
\text { to Afatinib; reduced cancer cell } \\
\text { migration }\end{array}$ & $\begin{array}{l}\text { Caco-2, Colorectal } \\
\text { cancer cell line }\end{array}$ & $\begin{array}{l}\text { Nanobiotechnol } \\
\text { et al. }{ }^{122}\end{array}$ \\
\hline 17 & $\begin{array}{l}\text { PEI-PDHA PEG-PDHA }{ }^{a} \text { [PEI-PDHA/ } \\
\text { PEG-PDHA/PTX/siSna/siTwi] (PPSTs) } \\
\text { nanoparticles }\end{array}$ & $\begin{array}{l}\text { Loaded with Twist1 } \\
\text { siRNA, Snail siRNA and } \\
\text { Paclitaxel }\end{array}$ & $\begin{array}{l}\text { Reduction of metastasis; inhibition of } \\
\text { cell invasion and ECM degradation }\end{array}$ & $\begin{array}{l}4 \mathrm{~T} 1 \text { mouse breast } \\
\text { cancer cell line; } \\
\text { female } \mathrm{BALB} / c \text { nude } \\
\text { mice }\end{array}$ & Tang et al. ${ }^{124}$ \\
\hline
\end{tabular}

${ }^{a}$ PEG - poly-ethylene glycol; PCL - peptide-poly-caprolactone; NSG - NOD. Cg-Prkdc ${ }^{\text {scid }}$ Il2 $2 \mathrm{rg}^{\mathrm{tm} 1 \mathrm{Wjl}} / \mathrm{SzJ}$; CPP - cell penetrating peptides; TNBC tripe negative breast cancer; NSCLC - non small cell lung cancer; PEI-PDHA - polyethyleneimine-block-poly[(1,4-butanediol)-dia-crylate- $b$-5hydroxyamylamine]; PEG-PDHA - polyethylene glycol-block-poly[(1,4-butanediol)-dia-crylate- $b$-5-hydroxyamylamine].

They are the only FDA (Food and Drug Administration) approved metal oxide nanoparticles to be used in magnetic resonance imaging (MRI). They have been successfully used in breast cancer detection and have also been reported to be used in the MRI-detection of the EMT state in breast cancer cells and pancreatic cancer cells, (reviewed by Malekigorji et al. and Thoidingjam et al.). ${ }^{69,70}$

\section{Hybrid nanocomposites}

Hybrid nanocomposites are the amalgamation of two or more elements (inorganic and organic) into one structure, exhibiting superior properties than their individual counterparts.

Hybrid nanocomposites are classified into two types (i) inorganic materials modified by organic moieties (organicsin-inorganics) and (ii) organic materials or matrices modified by inorganic moieties (inorganics-in-organics). ${ }^{71}$

In the former class, the inorganic structures or the colloidal particles are stabilized by the organic molecules. The later part depicts the functionalization of organic materials by inorganic constituents.

The inorganic constituents are metals, semiconductors, minerals, salts, non-metallic elements and their derivatives, etc. The organic matrices are either chemical (synthetic) or biological derivatives. The chemical (synthetic) molecules comprise of vehicles, coatings and capsules assembled into polymers, hydrogels, etc. whereas, the biological molecules encompass biomolecules such as lipids, enzymes, polysaccharides, proteins, nucleic acids, along with pharmaceutical substances, cells and microorganisms (bacteria). ${ }^{71}$

Hybrid nanoparticles are integrated with exceptional qualities such as, enhanced optical, magnetic and chemical properties that are designed to achieve dispersibility, targeting and responsiveness. For example, synthetic polymers such as PEG can be introduced to increase the circulation and retention time in vivo. Also, the introduction of targeting molecules (hyaluronic acid and folic acid) improves the targeting efficacy of the nanocomposite along with the enhanced permeability and retention effect (EPR). Altogether, these hybrid nanostructures are brought together by specific interactions that result in the synergistic enhancement of their functional properties. ${ }^{72}$

A study conducted by Tingting $\mathrm{Xu}$ et al. depicted that organic-inorganic hybrid nanocomposites based on chitosan derivatives (chitosan-glutathione, chitosan-glutathione-valine and chitosan-glutathione-valine-valine) and layered double hydroxides $(\mathrm{LDH})$ were successfully able to target peptide transporter-1 on the corneal epithelial cells in rabbits. These nanocomposites exhibited prolonged pre-corneal retention, superior corneal permeability and enhanced ocular bioavailability of the model drug pirenoxine sodium. Thus, these hybrid nanocomposites represent to be a promising drug delivery system for treating ocular diseases of the mid-posterior segments. $^{73}$

O. L. Galkina and co-workers designed an efficient drug delivery system based on cellulose nanofiber-titania nanocomposites and grafted it with three types of drugs (diclofenac sodium, penicillamine-D and phosphomycin). They observed that the various interactions between the nanocomposite and the drug molecules could be used to control the kinetics of long term drug release in vitro. Their study indicates the utilization of the nanocomposites in transdermal delivery of anaesthetics, analgesics and antibiotics. ${ }^{74}$

The various advantages of the nanocomposites in immunotherapy are reviewed in a study by Mingqiang Hao et al. that depicts their successful delivering strategy with numerous effects on the tumour microenvironment. ${ }^{75}$ 
A study conducted by Guibin Pand et al. revealed that APS-Au nanocomposites exhibit remarkable capability of inducing the maturation of dendritic cells and promoted the proliferation of T-cells. They also exhibit enhanced cytotoxicity against 4T1 tumour cells. Thus, their strong capability to inhibit tumour progression and pulmonary metastasis in vivo is portrayed. ${ }^{76}$

Ravi Prakash Shukla et al. constructed a spermine (SPM) tethered lipo-polymeric hybrid nanoconstructs having dual strategies. First, cell surface modification by heparin sulfate proteoglycans (HSPG) enhanced the specificity for intracellular internalization via receptor-mediated endocytosis. Second, $\mathrm{pH}$ dependant charge reversal in the tumour microenvironment facilitated the release of doxorubicin and genistein in a synergistic combination. This resulted in the induction of apoptosis, antiangiogenic effects upon inhibition of the VEGF pathway and inhibition of proliferation, invasion and metastasis in both in vitro and in vivo studies. ${ }^{77}$

\section{carbon-based nanoparticles}

Carbon nanoparticles have excellent biocompatibility, nonimmunogenicity, high loading efficiency, stability, optical properties and low toxicity. They exhibit the potential of drug loading through hydrophobic interactions or $\pi-\pi$ stacking (due to their pie electron conjugated surface) and their high surface area to mass ratio, thereby making them an efficient drug delivery vehicle. Utilizing their high fluorescence properties, they are conjugated to a ligand specific to an overexpressed receptor in cancer cells, for imaging and drug delivery. Among the numerous carbon nanomaterials, graphene, carbon nanotubes and carbon quantum dots are widely used for cancer therapy. ${ }^{78}$

The classification of carbon based nanoparticles is highly obscure. Thus, considering it as an individual entity, this review highlights the advantages of these nanoparticles in drug delivery, with an aim to inhibit metastasis.

Carbon nanoparticles possess the unique ability to enter into the nucleus of cancer stem cells, which contributes to their significance as a nano-drug delivery system. ${ }^{79}$ Besides, various carbon derived NPs have exhibited the properties of passively seeping into the tumour microenvironment, thereby inhibiting multiple factors such as CSCs, hypoxia, metastasis and invasion, angiogenesis, etc. (reviewed by Saleem et al.). Carbon dots downregulate the expression and activity of MMP2 and MMP9, therefore, migration, invasion and proliferation of lung adenocarcinoma (NSCLC) in vitro are inhibited ${ }^{80}$ they also inhibit ARF by binding to phosphor-tyrosine in prostate cancer cell lines. ${ }^{81}$

The ease of functionalization, surface modification and conjugation of the carbon derived nanoparticles render them to be a potential candidate as a drug delivery system in the tumour microenvironment with high efficacy. Functionalized carbon nanotubes (CNTs) are among the most stable forms of carbon nanoparticles with high drug loading efficiency, greater entrapment efficiency, improved bioavailability and pharmacokinetics of the free drugs in comparison to liposomes, dendrimers and inorganic nanoparticles. ${ }^{82}$
Multi-walled CNTs conjugated with folic acid and hyaluronic acid loaded with chemotherapeutic drugs (gemcitabine and doxorubicin) have been used efficiently in targeting breast and lung cancer cells, respectively. ${ }^{83,84}$ Under the guidance of a magnetic field, gemcitabine loaded hydrophilic multi-walled carbon nanotubes (mMWNTs), functionalized with magnetite nanoparticles and magnetic-activated carbon particles (mACs), exhibit high anti-tumour activity in vitro. It also resulted in regression and inhibition of lymph node metastasis, therefore, holding efficient therapeutic potential. ${ }^{85,86}$ A complex made up of chitosan-coated single-wall carbon nanotubes (SWNTs), loaded with salinomycin and functionalized with hyaluronic acid, resulted in the elimination of gastric cancer stem cells. Therefore, the self-renewal capacity is inhibited along with the decreased migration and invasion, ${ }^{87}$ enabling it to be a successful anti-CSC drug delivering system.

Gadolinium metallofullerenol nanoparticles, a derivative of fullerene nanoparticles (f-NPs), have shown to inhibit metastasis and invasion by inhibiting the MMPs in malignant breast cancer both in vitro and in vivo. ${ }^{88}$ They also exhibit inhibitory activity against Triple negative breast cancer (TNBC) cells due to the abrogation of breast cancer stem cells. They have also shown to block the TGF $\beta$ and hypoxia pathway, thereby eliminating tumour growth, EMT and metastasis. ${ }^{89}$

\section{Polymeric nanoparticles}

\section{Chitosan}

Chitosan is a naturally occurring polysaccharide, made up of chitin. It is biodegradable, biocompatible, cationic, highly basic and a mucoadhesive polymer, which is approved by the U.S. FDA for tissue engineering and drug delivery, (reviewed by Mohammed et al.)..$^{90}$

Chitosan nanoparticles (Ch-NPs) were successfully used to abrogate metastasis in esophageal cancer associated CAF (Cancer Associated Fibroblast) cell lines by downregulating the expression of matrix metalloproteases (MMP1 and MMP9) and chemokines (CXCR4, CXCR7, CCR5 and SDF1). ${ }^{91}$ Ch-NP functionalized with RGD peptide-loaded with Raloxifene abrogated tumorigenesis by inhibiting migration and angiogenesis in breast cancer cell lines. ${ }^{92}$ Haibo et al. observed that a novel chitosan-gemcitabine microsphere loaded with cisplatin was successful in the abrogation of EMT and metastasis of pancreatic cancer cells in vivo. ${ }^{93}$

Chitosan NPs have been a topic of extensive research to utilize their siRNA mediated gene silencing efficacy in single or dual therapies. Ch-NPs loaded with Twist siRNA were successfully able to induce sensitivity of the nasopharyngeal carcinoma cells to irradiation via MAPK downregulation; it also resulted in the ERK activation, which induced cell apoptosis. ${ }^{94}$ Snail siRNA and doxorubicin encapsulated carboxymethyl dextran (CMD) chitosan nanoparticles (dual drug/siRNA delivery system) have shown to exhibit the anti-tumour effect by inducing apoptosis and inhibiting EMT (by downregulation of MMP-9 and Vimentin and up-regulation of E-cadherin) in colorectal cancer cells ${ }^{95}$ and 
lung cancer cells ${ }^{96}$ in vitro. Similarly, chitosan nanoparticles loaded Snail siRNA and SN-38 (topo-1 inhibitor) resulted in the induction of E-cadherin and Claudin-1 expression, therefore, repressing EMT and metastasis in prostate cancer. ${ }^{97}$

\section{Micelles}

Polymeric micelles (PMs) are formed by the spontaneous arrangement of amphiphilic block copolymers in aqueous solution. They have a hydrophobic core and a hydrophilic shell that facilitate the loading of hydrophobic drugs in them. Their size ranges from 10 to100 nm, which passively accumulates at the tumour site via the EPR (enhanced permeability and retention) effect. The most commonly used hydrophilic segment of micelles used in drug delivery is made up of PEG, which minimises the non-specific interaction with the blood component and prolongs the circulation time, (reviewed by Zhang et al.). ${ }^{98}$

The polycation micelle is a widely used non-viral vector for drugs and siRNA delivery, due to their tenability and high transfection rate. A research conducted by Fang et al. showed that PEG-PLL-PLLeu micelles were capable of co-delivering ZEB siRNA and doxorubicin in cancer cells, resulting in the efficient knockdown the target genes, both in vitro and in vivo, leading to reduced EMT and repressed CSC properties, along with reduced metastasis in the lungs. ${ }^{99}$

Polymeric micelles prepared using Pluronic F127 (PM) and encapsulated with Salinomycin have been reported to interfere with the oncogenic protein vimentin and downregulates its expression, thus decreasing metastasis and inhibiting tumorigenesis in A549 lung adenocarcinoma cells. ${ }^{100}$

\section{PLGA nanoparticles}

Poly(lactic-co-glycolic acid), PLGA, is known to be among the best characterized biodegradable copolymer that decomposes into water and carbon dioxide molecules and is easily eliminated from the body, (reviewed by Rezvantalab et al.). ${ }^{101}$ Salinomycin loaded PLGA nanoparticles were found to be associated with the up-regulation of E-cadherin, $\beta$-catenin and TGF $\beta$ receptor expression in orthotopic tumours, which further resulted in the downregulation of EMT and tumour progression of pancreatic cancer. $^{102}$

PLGA based delivery of siRNA against DCAMKL1 (a novel intestinal and pancreatic stem cell marker) resulted in tumour growth arrest, inhibition of EMT and downregulation of c-Myc and Notch-1 via let-7a and miR-144 miRNA-dependent mechanisms, respectively, both in vitro and in vivo. ${ }^{103}$ Delivery of siRNA against NogoB receptors (regulates angiogenesis, vascular development and EMT in cancer cells) encapsulated into PLGA-PEI [poly(D,Llactide-co-glycolide)-2polyethylenimine] micelles conjugated with DMMA (2,3-dimethyl maleic anhydride) molecules exhibited a remarkable decrease in metastasis by normalizing tumour vessels and suppressing the EMT of breast cancer cells in mice bearing orthotopic breast carcinoma. ${ }^{104}$

A-Mangostin (naturally occurring xanthonoid) encapsulatedPLGA nanoparticles inhibited EMT by up-regulating E-cadherin and inhibiting N-cadherin, Slug, ZEB, the components of the sonic hedgehog pathway and stem cells markers, resulting in the inhibition of pancreatic neoplasia, pancreatic adenocarcinoma and liver metastasis in mice models. ${ }^{105}$

Similarly, anthothecol (naturally occurring limonoid, used as an anti-malarial compound) encapsulated PLGA nanoparticles inhibit the sonic hedgehog pathway, thereby suppressing cell motility, migration and invasion in pancreatic cancer. ${ }^{106}$

Wedelolactone (naturally occurring coumestan) encapsulated PLGA nanoparticles exhibited an increased uptake in triple negative breast cancer cells and CSC populations, with enhanced drug retention due to the downregulation of SOX2 and ABCG2 genes, preventing EMT. In combination with paclitaxel, a reduced population of stem cells was observed, in addition to the decreased metastasis and enhanced chemosensitivity. ${ }^{107}$

\section{Naturally occurring biomembranes}

With the growing popularity of nanoparticles for their exceptional drug loading capacity in the treatment of various malignancies, there has been tremendous debate about their long term toxicity or any other side effects in our body. In order to curb this debate, researchers came up with the idea of using naturally occurring multi-vesicular bodies (liposomes and exosomes) which intrinsically possess drug loading capabilities. However, very few research studies have been conducted to date, with promising results depicting their probable roles in abrogating tumorigenesis.

Liposomes. Liposomes are amiphipathic molecules consisting of single or multiple phospholipid bilayer membranes that are formed by the hydrophobic and hydrophilic interactions with the aqueous phase. Their constituent lipid molecules render them with exceptional characteristics such as biocompatibility, biodegradability and are metabolized with limited toxicity in our body. Due to their constituent lipid bilayer, they are used as drug delivery vehicles for both hydrophilic and hydrophobic drugs. ${ }^{126}$ Moreover, the surface of the liposomes can be functionalised using various ligands, sugars and other moieties to allow specific uptake by the tumours. ${ }^{127}$ Studies indicate that liposomes can enter the tumour through the enhanced permeability and retention (EPR) effect that prolongs the drug's retention time in the body, inhibits degradation of the entrapped chemotherapeutic agents, and accumulates at the tumour site with minimal systemic toxicity. ${ }^{128}$ Due to their resemblance to biomembranes, they provide protection against oxidative stress and have no signs of necrosis or inflammation in healthy tissues. ${ }^{129}$ They are known to play an essential role in curbing EMT and metastasis, upon functionalization with numerous ligands and encapsulation with various chemotherapeutic agents.

PEGylated liposomes encapsulating a radiopharmaceutical, Rhenium-188 (188Re) have been reported to inhibit proliferation and EMT of human head and neck cancer cells in vivo. The 188Re-liposomes are thought to induce the expression of Let-7 miRNA, which is known to inhibit EMT by suppressing the HMGA2 (high mobility group AT hook2) gene that activates the expression of SNAIL and Twist, thus, inhibiting tumour growth and metastasis. ${ }^{130}$ The intraperitoneal delivery of 188ReLiposome has shown to switch the metabolism from glycolysis to oxidative phosphorylation, inhibit EMT and reactivate the 
function of p53, thereby representing a novel treatment for ovarian cancer. ${ }^{131}$

Delivery of various STAT3 inhibitors, encapsulated in the liposomes, has exhibited profound effects on tumorigenesis such as elimination of tumours by decreasing STAT3 mediated immune suppression, reduction in proliferation, and immune modulation to eliminate tumours, (reviewed by Kullberg et al.). ADH-1 modified liposomes facilitate the delivery of paclitaxel and other fluorescent probes into the tumour cells and have shown to restore chemosensitivity and suppress migration by targeting EMT. This nanocarrier was highly efficient in reducing tumorigenesis and had exceptional tumour targeting ability. ${ }^{132}$

Nanoliposomal Quercetin (nLQ), possessing cytomembrane permeability, was shown to downregulate NF- $\mathrm{BB}, \mathrm{p} 65$, HDAC1 and Cyclin D1and upregulate caspase3 in epithelial cancer cells. Also, there was an enhanced expression of E-cadherin, which demonstrated the downregulation of EMT. In combination with CD133 antiserum, it exhibited an enhanced apoptotic effect that contributed to the apoptosis of the cancer stem cells. ${ }^{133}$

Dioscin liposomes in combination with GGP (a cell penetrating peptide consisting of eight amino acids Gly-Gly-Pro-PheVal-Tyr-Leu-Ile) modified daunorubicin inhibit breast cancer by suppressing EMT both in vitro and in vivo. Dioscin especially downregulates VEGF, MMP9, N-cadherin and vimentin, thereby suppressing EMT.

Liposomes loaded with PLD inhibitors (5-fluoro-2-indolyldeschlorohalopemide, FIPI) in combination with a commercially available anti-cancer drug, doxorubicin and functionalized with D-alpha tocopheryl acid succinate ( $\alpha$-TOS) that provides negative charge to the liposomal surface, [briefly, liposomes containing DOX, FIPI, and $\alpha$-TOS (DFT-lip)] were found to be effective in inhibiting motility, migration and invasion in highly metastatic breast cancer in vitro and in vivo. Besides, they also inhibited proliferation and induced apoptosis.

The liposome formulation based on simvastatin (which disrupts lipid raft and suppress integrin $\beta 3$ and focal adhesion formulation) was reported to inhibit the FAK signalling pathway, repolarize the tumour associated macrophages (TAM), remodel the tumour microenvironment and suppress the EMT. It also resensitized cancer cells to paclitaxel treatment. ${ }^{134}$

There are several types of liposomes depending on their size, lamellar number and formulation of constitutes. Liposomes show numerous advantages over conventional chemotherapy and their specific properties make them more effective as a drug delivery vehicle. Recent advancement in the study of liposomes illustrated the great potential for their widespread adoption in cancer treatment.

Exosomes. In general, exosomes carry biologically active molecules like proteins and nucleic acids that regulate gene expression and cellular functions in the targeted cells across the circulation and act as intercellular communicators. They are formed in the inter-luminal vessels (ILVs) of the multivesicular bodies (MVBs) which fuse with the plasma membrane and are released in the extracellular space by the process of exocytosis. The Rab GTPase family of proteins is involved in the fusion of the MVBs with the plasma membrane resulting in the formation of exosomes. Endosomal sorting complex required for transport (ESCRT) is the principal molecular machinery which coordinates the formation of exosomes.

Exosomes are released under normal physiological conditions as well as under abnormal conditions like in case of cancer. Studies have shown that the magnitude of the release of exosomes increases in the case of diseased conditions. The cellular origin of exosomes plays a vital role in the characteristics of the cargoes that it carries. By taking advantage of the unique characteristics of exosomes, they can be functionalized as well as loaded with different drugs or small molecules to combat EMT, as illustrated in Fig. 5. Since exosomes are isolated from the patient's own cells, they do not elicit an immune response, and due to their nanosize, they can easily penetrate the cells and deliver the payload into the recipient cells. Thus, exosomes have been proved to be a desirable option as a drug delivery vehicle for cancer treatment. ${ }^{135}$

The treatment strategies involving exosomes were successfully observed by Lydia and co-workers, upon intravenously injecting

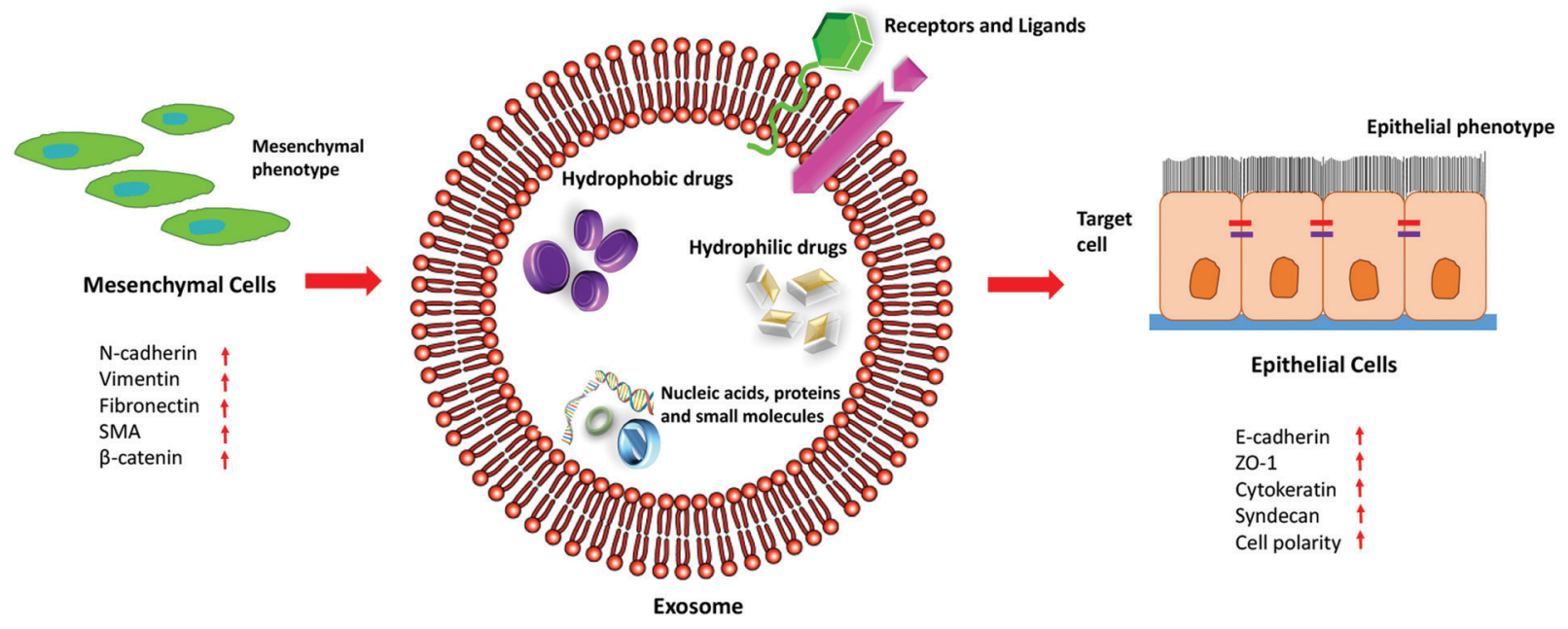

Fig. 5 The therapeutic efficacy of exosomes in EMT. 
neuron-specific RVG-peptide-functionalized exosomes, loaded with GAPDH siRNA, specifically targeting microglia, neurons, oligodendrocytes in the brain resulting in the knockdown of BACE1 gene, a therapeutic target in Alzheimer's disease. ${ }^{136}$ Akhil et al. assessed the therapeutic effects of doxorubicin loaded exosomes in nonsmall cell lung cancer in vitro under acidic conditions and were successful in demonstrating their efficient uptake and cytotoxicity in cancer cells with minimal effects in non-cancerous cells. ${ }^{137}$

Later on, a study conducted using two prostate cancer cell lines, PC-3 (highly metastatic with tumorigenic potential) and LNCaP (less metastatic), indicated the tumour suppressive role of miR-26a in prostate cancer cells. The proteomic profile of the exosomes from these cell lines showed that LNCaP had a higher level of miR-26a as compared to PC-3. When PC-3 cells were co-cultured with LNCap exosomes, there was a decrease in the metastatic and tumorigenic capacities of the prostate cancer cells in vitro. ${ }^{138}$

Exosomes derived from bone marrow mesenchymal stem cells (hBMSCs) containing miR-101 were able to inhibit EMT in oral cancer cell lines. COL10A1 is upregulated in oral cancer cell lines. Exosomes carrying miR-101 from hBMSCs when inserted in oral cancer cells inhibited COL10A1, which resulted in the inhibition of proliferation, invasion and migration of these cells. ${ }^{139}$ miR-101 inhibits EMT progression by directly targeting the specific binding sites on the 3 '-UTRs of ZEB-1 and ZEB-2, which are suppressors of E-cadherin. ${ }^{140}$

microRNA-148b is found to be lower in CAFs as compared to normal fibroblasts. In a study done by Li et al., when exosomes derived from miR-148b transfected CAFs were used to treat endometrial cell lines, there was a decrease in cell migration and invasion. DNA methyltransferase 1 (DNMT1), a downstream target gene which is involved in various cancer progression, was directly targeted by miR-148b, resulting in the repression of EMT. ${ }^{141,142}$ A non-coding RNA miR-30e is highly downregulated in cholangiocarcinoma (CCA) cells. It is targeted by TGF- $\beta$ which is further predicted to target Snail, overexpressed during EMT. When miR-30e containing exosomes were used to treat CCA cells, there was suppression in cell invasion and migration, along with EMT inhibition. ${ }^{143}$

TRIM59 gene is a tumour promoting factor in breast cancer cells, which modulates the PDCD10 and results in the suppression of its associated signalling pathway, ROCK $1 .{ }^{144} \mathrm{miR}-148 \mathrm{~b}-3 \mathrm{p}$ is poorly expressed in breast cancer tissues as studied by Yuan., et al. Human umbilical cord mesenchymal stem cell (HUCMSC) derived exosomes containing miR-148b-3p, when co-cultured with MDA MB-231 cell lines, induced downregulation of TRIM 59 gene expression, thereby suppressing the progression of breast cancer and inhibiting EMT. ${ }^{145}$

Therefore, this multi-vesicular body, due to its size, origin and similarity with synthetic liposomes has gained much importance to be used as a drug delivery agent. Its innate ability renders it to be a suitable nanocarrier for the delivery of chemotherapeutic drugs, genes or recombinant proteins into the targeted cells or tumour microenvironment. Additionally, exosomes enhance the specific targeting ability of tumour cells through the surface receptors expressed on their membranes.
Numerous studies have established the eminence of treatment with exosome-mediated delivery of miRNAs or drugs to suppress tumour progression. Therefore, better therapeutic significance can be achieved by following the co-therapeutic regime, which would synergistically regulate the EMT and can be employed for future studies. Moreover, further research is required in understanding the various exosome-mediated crosstalks in malignancies which would render deeper insights into the utilization of exosomal cargoes, based on their diagnostic and therapeutic potentials.

\section{Conclusions}

With the increasing knowledge of EMT and its association with numerous tumorigenic properties, it has been a necessity to curb the phenomenon by blocking the pathways associated with it or by inhibiting the molecules that enhance this phenomenon. The reasonable possibilities of blocking the entire invasion process and restoring normal homeostasis render the initial step of the cascade an attractive target of research. Since EMT is associated with the tumour microenvironment, it has been a complex challenge to discover the method of delivering drugs/inhibitors inside the tumour cells for a significant therapy regime. Nanoparticles bestow us with the efficient, targeted delivery of drugs inside the tumours with enhanced efficacy. It also allows the delivery of other small molecular inhibitors including the oligonucleotides and peptides, which otherwise are susceptible to degradation inside the body.

Metallic nanoparticles (gold) have shown tremendous promising results in the inhibition of metastasis and invasion due to their intrinsic cytotoxic ability. However, the use of metal and metal oxide nanoparticles, despite their anti-tumorigenic properties, exhibits cytotoxic effects when used for a prolonged period of time.

Hybrid nanocomposites exhibit enhanced physical and chemical properties than their individual counterparts. Hybrid nanostructures are potent mimics of natural structures. Thus, they are superior for targeting numerous functions and can be used as an efficient drug delivery vehicle.

Carbon-based nanostructures endue enhanced drug loading efficacy, photo-stability and easy functionalization with good photoluminescence and water solubility. The surface of carbon nanotubes can be customised with molecules of choice by adsorption, electrostatic interactions or covalent bonding that renders them hydrophilic. Moreover, the fluorescence properties exhibited by the carbon quantum dots make them suitable for bioimaging applications. But their innate ability to induce tumorigenesis in some cases limits their usage.

The extraordinary characteristics of polymeric nanoparticles are the controlled release of therapeutic agents. While synthetic polymeric NPs include easy fabrication and absence of biological contamination, polycationic polymers (chitosan) possess better mucoadhesive properties, which render them soluble in acidic pH. Additionally, the biodegradable PLGA NPs are initially hydrolysed into its monomeric units, lactic acid and 
glycolic acid, a by-product of various metabolic pathways under normal physiological conditions, which are further degraded into water and carbon dioxide molecules inside our body. On the other hand, chitosan is degraded into non-toxic oligosaccharide, contributing to the efficient delivery of drugs without posing cytotoxicity.

As a matter of fact, the use of nanoparticles has been a constant topic of debate due to the inadequacy of dosage requirement and their side effects in both healthy and tumour cells, which requires arduous research before implementing them in the therapeutic regime. Therefore, researchers have come up with naturally occurring multi-vesicular bodies (liposomes and exosomes) as an alternative to the above-mentioned nanoparticles. The resemblance of liposomes to biomembranes renders protection against oxidative stress and inflammation in healthy cells. However, their facile cellular uptake due to their outer lipid bilayer serves as an added advantage. On the other hand, the inherent nature of exosomes to carry cargoes, the presence of various adhesion molecules on the surface of the exosomes and their ability to fuse with the cell membrane make them suitable to serve as a drug-delivering vehicle, with increased efficiency and specificity to the tumour cells. Furthermore, exosomes are similar in composition to the body's own cells; thus, they are non-immunogenic in nature which serves as a significant advantage.

Exosomes are one of the highly researched extracellular vesicles. They are derived from different cells, having extensive roles and targets, and can be isolated using wide varieties of techniques. ${ }^{146}$ Since they are native to our body, they avoid phagocytosis and lysosomal degradation. Exosomes also exhibit enhanced stability in the blood, allowing them to travel long distances in the body under normal physiological and extreme pathological conditions. Their intrinsic homing characteristics endow them with the uniqueness of being the most suitable and efficient drug delivery system into the tumour microenvironment.

Thus, owing to the increasing interest and requisite development of novel therapies for oncologic patients, prevention of systemic dissemination of the malignancies remains a consistent aim. Besides the various advantages, the long term cytotoxicity associated with the nanoparticles is of greatest concern and the evaluation to a sub-toxic dose of a nanoparticle remains a considerable challenge. This review sheds light on the different types of nanoparticles that can be used in order to achieve a successful targeted delivery. However, recent advances in the utilization of biomembranes, especially exosomes, as a drug carrier are incredible. Thus, advanced research is required to ascertain the applications of these nanomaterials as a drug loading vehicle which might provide us with a better therapeutic approach.

Hence, the reviewed data suggest nanotechnology as a promising tool for the modulation, counteracting and efficient treatment for the metastatic process in various malignancies.

\section{Conflicts of interest}

The authors declare no conflict of interest.

\section{Acknowledgements}

We acknowledge the support of the Department of Biotechnology, Government of India (BT/PR13560/COE/34/44/2015 and BT/PR 25095/NER/95/1011/2017). The authors also acknowledge the DBT Programme Support Project, Central Instruments Facility (CIF), Centre for Nanotechnology at IIT Guwahati.

\section{References}

1 M. Boareto, M. K. Jolly, A. Goldman and H. Levine, NotchJagged signalling can give rise to clusters of cells exhibiting a hybrid epithelial/mesenchymal phenotype, J. R. Soc. Interface, 2016, 13, 20151106.

2 F. Portillo and A. Cano, Transcriptional regulation of cell polarity in EMT and cancer, Oncogene, 2008, 6958-6969, DOI: 10.1038/onc.2008.346.

3 C. Min, S. F. Eddy, D. H. Sherr and G. E. Sonenshein, NF-kB and Epithelial to Mesenchymal Transition of Cancer, J. Cell Biochem., 2008, 744, 733-744.

4 S. Terry, et al., New insights into the role of EMT in tumor immune escape, Mol. Oncol., 2017, 11, 824-846.

5 Z. Cao, T. Livas and N. Kyprianou, Anoikis and EMT: Lethal "Liaisons" during Cancer Progression, Crit. Rev. Oncog., 2016, 21(3-4), 155-168.

6 S. Lamouille, J. Xu and R. Derynck, Fakultas Psikologi Dan Sosial Budaya Universitas Islam Indonesia Yogyakarta, Nat. Rev. Mol. Cell Biol., 2014, 15, 178-196.

7 J. Haynes, J. Srivastava, N. Madson, T. Wittmann and D. L. Barber, Dynamic actin remodeling during epithelial-mesenchymal transition depends on increased moesin expression, Mol. Biol. Cell, 2011, 22, 4750-4764.

8 J. Shankar, et al., Pseudopodial actin dynamics control epithelial-mesenchymal transition in metastatic cancer cells, Cancer Res., 2010, 70, 3780-3790.

9 C. M. Fife, J. A. McCarroll and M. Kavallaris, Movers and shakers: Cell cytoskeleton in cancer metastasis, $\mathrm{Br}$. J. Pharmacol., 2014, 171, 5507-5523.

10 B. Sun, Y. Fang, Z. Li, Z. Chen and J. Xiang, Role of cellular cytoskeleton in epithelial-mesenchymal transition process during cancer progression, Biomed. Rep., 2015, 3, 603-610.

11 V. M. Golubovskaya, Targeting FAK in human cancer: From finding to first clinical trials, Front. Biosci., 2014, 19, 687-706.

12 M. L. Ackland, et al., Epidermal growth factor-induced epithelio-mesenchymal transition in human breast carcinoma cells, Lab. Investig., 2003, 83, 435-448.

13 F. Strutz, et al., Role of basic fibroblast growth factor-2 in epithelial-mesenchymal transformation, Kidney Int., 2002, 61, 1714-1728.

$14 \mathrm{~J}$. Farrell, et al., HGF induces epithelial-to-mesenchymal transition by modulating the mammalian Hippo/MST2 and ISG15 pathways, J. Proteome Res., 2014, 13, 2874-2886.

$15 \mathrm{Q} . \mathrm{Wu}$, et al., Emerging roles of PDGF-D in EMT progression during tumorigenesis, Cancer Treat. Rev., 2013, 39, 640-646. 
16 M. Natsuizaka, et al., Interplay between Notch1 and Notch3 promotes EMT and tumor initiation in squamous cell carcinoma, Nat. Commun., 2017, 8, 1758.

17 J. Chen, N. Imanaka, J. Chen and J. D. Griffin, Hypoxia potentiates Notch signaling in breast cancer leading to decreased E-cadherin expression and increased cell migration and invasion, Br. J. Cancer, 2010, 351-360, DOI: 10.1038/sj.bjc.6605486.

18 Y. G. Jiang, et al., Role of Wnt/ $\beta$-catenin signaling pathway in epithelial-mesenchymal transition of human prostate cancer induced by hypoxia-inducible factor-1 $\alpha$, Int. J. Urol., 2007, 14, 1034-1039.

19 Y. Cao, et al., ScienceDirect Implications of the Notch1Snail/Slug-epithelial to mesenchymal transition axis for lymph node metastasis in infiltrating ductal carcinoma, Kaohsiung J. Med. Sci., 2015, 31, 70-76.

20 T. Gui, Y. Sun, A. Shimokado and Y. Muragaki, The Roles of Mitogen-Activated Protein Kinase Pathways in TGF$\beta$-Induced Epithelial-Mesenchymal Transition, J. Signal Transduct., 2012, 2012, 1-10.

21 F.-F. Hsu, Myocardial Extraction from Newborn Rats HHS Public Access, Physiol. Behav., 2016, 176, 139-148.

$22 \mathrm{M}$. H. Yang and K. J. Wu, TWIST activation by hypoxia inducible factor-1 (HIF-1): Implications in metastasis and development, Cell Cycle, 2008, 7, 2090-2096.

23 M. Suarez-Carmona, J. Lesage, D. Cataldo and C. Gilles, EMT and inflammation: inseparable actors of cancer progression, Mol. Oncol., 2017, 11, 805-823.

24 J. R. M. Sophie Mokas, et al., Uncoupling Stress Granule Assembly and Translation Initiation Inhibition, Mol. Biol. Cell, 2009, 20, 2673-2683.

25 S. Shao, et al., Notch1 signaling regulates the epithelial mesenchymal transition and invasion of breast cancer in a Slug-dependent manner, Mol. Cancer, 2015, 1-17, DOI: 10.1186/s12943-015-0295-3.

26 Y. Wang, J. Shi, K. Chai, X. Ying and B. Zhou, The Role of Snail in EMT and Tumorigenesis, Curr. Cancer Drug Targets, 2014, 13, 963-972.

27 M. Jordà, et al., Upregulation of MMP-9 in MDCK epithelial cell line in response to expression of the Snail transcription factor, J. Cell Sci., 2005, 118, 3371-3385.

$28 \mathrm{~J}$. Albanell, et al., A SNAIL1-SMAD3/4 transcriptional repressor complex promotes TGF-B mediated epithelialmesenchymal transition, Nat. Cell Biol., 2013, 11, 943-950.

29 M. H. Yang, et al., Bmi1 is essential in Twist1-induced epithelial-mesenchymal transition, Nat. Cell Biol., 2010, 12, 982-992.

30 G. A. Castillon, et al., Septins Have a Dual Role in Controlling Mitotic Exit in Budding Yeast We assayed the spindle position checkpoint in these mutants with movies of living cells progressing through mitosis. The cells expressed GFP-Tub1p, allowing us to, Curr. Biol., 2003, 13, 654-658.

31 A. A. Postigo, J. L. Depp, J. J. Taylor and K. L. Kroll, Regulation of Smad signaling through a differential recruitment of coactivators and corepressors by ZEB proteins, EMBO J., 2003, 22, 2453-2462.

32 N. Dave, et al., Functional cooperation between snail1 and twist in the regulation of ZEB1 expression during epithelial to mesenchymal transition, J. Biol. Chem., 2011, 286, 12024-12032.

33 M. Olea-Flores, et al., Extracellular-signal regulated kinase: A central molecule driving epithelial-mesenchymal transition in cancer, Int. J. Mol. Sci., 2019, 20, 1-32.

34 Z. Wang, Y. Li, D. Kong and F. H. Sarkar, The Role of Notch Signaling Pathway in Epithelial-Mesenchymal Transition (EMT) During Development and Tumor Aggressiveness, Curr. Drug Targets, 2010, 11, 745-751.

35 S. S. Islam, et al., Sonic hedgehog (Shh) signaling promotes tumorigenicity and stemness via activation of epithelial-tomesenchymal transition (EMT) in bladder cancer, Mol. Carcinog., 2016, 55, 537-551.

36 L. Larue and A. Bellacosa, Epithelial-mesenchymal transition in development and cancer: Role of phosphatidylinositol 3' kinase/AKT pathways, Oncogene, 2005, 24, 7443-7454.

37 Manuscript, A. NIH Public Access, 2010, 19, 128-139.

38 Y. Hao, D. Baker and P. Dijke, TGF- $\beta$-Mediated EpithelialMesenchymal Transition and Cancer Metastasis, Int. J. Mol. Sci., 2019, 20(11), 2767.

39 B. Fe, Exosomes: endosomal-derived vesicles shipping extracellular messages, Curr. Opin. Cell Biol., 2004, 16(4), 415-421.

40 L. J. Vella, The emerging role of exosomes in epithelial mesenchymal-transition in cancer, Front. Oncol., 2014, 4, 1-5.

41 A. Conigliaro and C. Cicchini, Exosome-Mediated Signaling in Epithelial to Mesenchymal Transition and Tumor Progression, J. Clin Med., 2019, 8(1), 26.

42 L. Chen, et al., HCC-derived exosomes elicit HCC progression and recurrence by epithelial- mesenchymal transition through MAPK/ERK signalling pathway, Cell Death Dis., 2018, 9, 513.

43 D. Xiao, et al., Cancer Lett., 2017, 376, 318-327.

$44 \mathrm{~S}$. He, et al., Exosomal miR-499a-5p promotes cell proliferation, migration and EMT via mTOR signaling pathway in lung adenocarcinoma, Exp. Cell Res., 2019, 379, 203-213.

45 W. Zhou, et al., NIH Public Access, 2015, 25, 501-515.

$46 \mathrm{~W}$. Li, et al., TGF $\beta 1$ in fibroblasts-derived exosomes promotes epithelial-mesenchymal transition of ovarian cancer cells, Oncotarget., 2017, 8, 96035-96047.

47 I. Pastushenko, et al., Identification of the tumour transition states occurring during EMT, Nature, 2018, 556, 463-468.

48 N. P. A. D. Gunasinghe, A. Wells, E. W. Thompson and H. J. Hugo, Mesenchymal-epithelial transition (MET) as a mechanism for metastatic colonisation in breast cancer, Cancer Metastasis Rev., 2012, 31, 469-478.

49 M. Lu, M. K. Jolly, H. Levine, J. N. Onuchic and E. BenJacob, MicroRNA-based regulation of epithelial-hybrid- 
mesenchymal fate determination, Proc. Natl. Acad. Sci. U. S. A., 2013, 110, 18144-18149.

50 M. K. Jolly, et al., Implications of the Hybrid Epithelial/ Mesenchymal Phenotype in Metastasis, Front. Oncol., 2015, 5, 155.

51 R. R. Arvizo, et al., Inhibition of tumor growth and metastasis by a self-therapeutic nanoparticle, Proc. Natl. Acad. Sci. U. S. A., 2013, 110, 6700-6705.

$52 \mathrm{~W}$. Yang and X. Yang, Gold nanoparticles attenuate metastasis by tumor vasculature normalization and epithelial mesenchymal transition inhibition, Int. J. Nanomed., 2017, 3509-3520.

53 X. Xiong, et al., Sensitization of ovarian cancer cells to cisplatin by gold nanoparticles, Oncotarget., 2014, 5(15), 6453-6465.

54 Y. Huai, Y. Zhang, X. Xiong, S. Das and R. Bhattacharya, Gold Nanoparticles sensitize pancreatic cancer cells to gemcitabine, Cell Stress, 2019, 3, 267-279.

55 G. N. Therapy and A. Cell, HHS Public Access., 2018, 12, 9279-9290.

56 N. Kumar, et al., Biomaterials Low doses of PEG-coated gold nanoparticles sensitize solid tumors to cold plasma by blocking the PI3K/AKT-driven signaling axis to suppress cellular transformation by inhibiting growth and EMT, Biomaterials, 2016, 87, 118-130.

57 S. B. F. A. Bhat, et al., Gold nanoparticle - conjugated quercetin inhibits epithelial - mesenchymal transition, angiogenesis and invasiveness via EGFR/VEGFR-mediated pathway in breast cancer, Cell Prolif., 2016, 678-697, DOI: 10.1111/cpr.12296.

58 Glucocorticoid receptor-mediated delivery of nano gold withaferin conjugates for reversal of epithelial-tomesenchymal transition and tumor regression, 2016.

59 S. A. Loutfy, et al., Anti-proliferative activities of metallic nanoparticles in an in vitro breast cancer model, Asian Pacific J. Cancer Prev., 2015, 16, 6039-6046.

60 S. N. Sunil Gowda, et al., Gallic acid-coated sliver nanoparticle alters the expression of radiation-induced epithelial-mesenchymal transition in non-small lung cancer cells, Toxicol. Vitr., 2018, 52, 170-177.

61 L. Vila, R. Marcos and A. Hernández, Long-term effects of silver nanoparticles in CaCo-2 cells, Nanotoxicology, 2017, 11(6), 771-780.

62 A. Khurana, S. Tekula, M. A. Saifi, P. Venkatesh and C. Godugu, Therapeutic applications of selenium nanoparticles, Biomed. Pharmacother., 2019, 111, 802-812.

63 M. Kumari, et al., Curcumin-loading potentiates the chemotherapeutic efficacy of selenium nanoparticles in HCT116 cells and Ehrlich' s ascites carcinoma bearing mice CSIR-Institute of Genomics and Integrative Biology, Delhi University Campus, Mall Road, Academy of Scien, Eur. J. Pharm. Biopharm., 2017, 117, 346-362.

64 M. Kumari, M. Prasad, S. Patnaik and Y. Shukla, European Journal of Pharmaceutics and Biopharmaceutics Curcumin loaded selenium nanoparticles synergize the anticancer potential of doxorubicin contained in self- assembled, cell receptor targeted nanoparticles, Eur. J. Pharm. Biopharm., 2018, 130, 185-199.

$65 \mathrm{X} . \mathrm{Li}$, et al., Inhibition of Epithelial - Mesenchymal Transition and Tissue Regeneration by Waterborne Titanium Dioxide Nanoparticles, ACS Appl. Mater. Interfaces, 2018, 10(4), 3449-3458.

66 F. Peng, J. K. Tee, M. I. Setyawati and X. Ding, Inorganic Nanomaterials as Highly Efficient Inhibitors of Cellular Hepatic Fibrosis, ACS Appl. Mater. Interfaces, 2018, 10(38), 31938-31946.

67 R. Wahab, N. Kaushik, F. Khan and N. K. Kaushik, SelfStyled ZnO Nanostructures Promotes the Cancer Cell Damage and Supresses the Epithelial Phenotype of Glioblastoma, Nat. Publ. Gr., 2016, 1-13, DOI: 10.1038/ srep19950.

68 Y. Wang, et al., Cuprous oxide nanoparticles inhibit the growth and metastasis of melanoma by targeting mitochondria, Cell Death Dis., 2013, 4, e783.

69 S. Thoidingjam and A. B. Tiku, New developments in breast cancer therapy: role of iron oxide nanoparticles, Adv. Nat. Sci., 2017, 8(2), 023002.

70 M. Malekigorji, A. D. M. Curtis and C. Hoskins, The Use of Iron Oxide Nanoparticles for Pancreatic Cancer Therapy, J. Nanomed. Res., 2014, 1, 1-12.

71 M. S. Saveleva, et al., Hierarchy of hybrid materials-the place of inorganics-in-organics in it, their composition and applications, Front. Chem., 2019, 7, 1-21.

72 V. P. Ananikov, Organic - Inorganic Hybrid Nanomaterials, Nanomaterials, 2019, 9(9), 1197.

73 T. Xu, J. Zhang, H. Chi and F. Cao, Multifunctional properties of organic-inorganic hybrid nanocomposites based on chitosan derivatives and layered double hydroxides for ocular drug delivery, Acta Biomater., 2016, 36, 152-163.

74 O. L. Galkina, V. K. Ivanov, A. V. Agafonov, G. A. Seisenbaeva and V. G. Kessler, Cellulose nanofibertitania nanocomposites as potential drug delivery systems for dermal applications, J. Mater. Chem. B, 2015, 3, 1688-1698.

75 M. Hao, B. Chen, X. Zhao, N. Zhao and F. Xu, Organic/ inorganic nanocomposites for cancer immunotherapy, Mater. Chem. Front., 2020, 4, 2571-2609.

76 G. Pang, et al., Immunoactive polysaccharide functionalized gold nanocomposites promote dendritic cell stimulation and antitumor effects, Nanomedicine, 2019, 14, 1291-1306.

77 R. P. Shukla, et al., Multifunctional hybrid nanoconstructs facilitate intracellular localization of doxorubicin and genistein to enhance apoptotic and anti-angiogenic efficacy in breast adenocarcinoma, Biomater. Sci., 2020, 8, 1298-1315.

78 J. Saleem, L. Wang and C. Chen, Carbon-Based Nanomaterials for Cancer Therapy via Targeting Tumor Microenvironment, Adv. Healthcare Mater., 2018, 1800525, 1-30.

79 W. Su, et al., Red-Emissive Carbon Quantum Dots for Nuclear Drug Delivery in Cancer Stem Cells, J. Phys. Chem. Lett., 2020, 11(4), 1357-1363.

80 Manuscript, A. rsc.li/njc, 2018, DOI: 10.1039/C8NJ02092B. 
81 Y. Xie, et al., HHS Public Access., 2019, 38, 2967-2983.

82 N. K. Mehra and N. K. Jain, Functionalized carbon nanotubes and their drug delivery applications, Nanostruct. Drug Delivery, 2014, 4, 327-329.

83 R. Singh, N. K. Mehra, V. Jain and N. K. Jain, Gemcitabineloaded smart carbon nanotubes for effective targeting to cancer cells, J. Drug Target., 2013, 21(6), 581-592.

84 S. R. Datir, M. Das, R. P. Singh and S. Jain, Hyaluronate Tethered, " Smart" Multiwalled Carbon Nanotubes for Tumor-Targeted Delivery of Doxorubicin, Bioconjugate Chem., 2012, 23(11), 2201-2213.

85 F. Yang, et al., Magnetic functionalised carbon nanotubes as drug vehicles for cancer lymph node metastasis treatment, Eur. J. Cancer, 2011, 7, 0-9.

86 D. Yang, F. Yang, J. Hu, J. Long and C. Wang, Hydrophilic multi-walled carbon nanotubes decorated with magnetite nanoparticles as lymphatic targeted drug delivery vehicles, Chem. Commun., 2009, 4447-4449, DOI: 10.1039/b908012k.

87 H. Yao, Y. Zhang, L. Sun and Y. Liu, Biomaterials The effect of hyaluronic acid functionalized carbon nanotubes loaded with salinomycin on gastric cancer stem cells, Biomaterials, 2014, 35, 9208-9223.

88 A. Manuscript NIH Public Access, 2012, 8, 136-146.

89 Y. Liu, et al., Breast cancer stem cell-specific inhibitor, Nat. Commun., 2015, 6, 1-18.

90 M. A. Mohammed, J. T. M. Syeda, K. M. Wasan and E. K. Wasan, An Overview of Chitosan Nanoparticles and Its Application in Non-Parenteral Drug Delivery, Pharmaceutics, 2017, 9(4), 53.

91 P. D. Potdar and A. U. Shetti, Evaluation of anti-metastatic effect of chitosan nanoparticles on esophageal cancerassociated fibroblasts, J. Cancer Metastasis Treat., 2016, 2, 259.

92 Manuscript, A. Nanoscale, 2020, DOI: 10.1039/C9NR10673A.

$93 \mathrm{H}$. Yu, et al., The effects of novel chitosan-targeted gemcitabine nanomedicine mediating cisplatin on epithelial mesenchymal transition, invasion and metastasis of pancreatic cancer cells, Biomed. Pharmacother., 2017, 96, 650-658.

$94 \mathrm{X}$. Zhuo, et al., Nanoparticle-mediated down-regulation of TWIST increases radiosensitivity of nasopharyngeal carcinoma cells via ERK pathway, Am. J. Cancer Res., 2015, 5, 1571-1579.

95 S. Sadreddini, et al., Chitosan nanoparticles as a dual drug/ siRNA delivery system for treatment of colorectal cancer, Immunol. Lett., 2017, 181, 79-86.

96 M. Kay, Cellular and Molecular Biology: Preface, Cell. Mol. Biol., 2005, 51, 117.

97 C. Houde, et al., Overexpression of the NOTCH ligand JAG2 in malignant plasma cells from multiple myeloma patients and cell lines, Blood, 2004, 104, 3697-3704.

98 Y. Zhang, Y. Huang and S. Li, Polymeric micelles: Nanocarriers for cancer-targeted drug delivery, AAPS PharmSciTech, 2014, 15, 862-871.

99 S. Fang, et al., ZEB1 knockdown mediated using polypeptide cationic micelles inhibits metastasis and effects sensitization to a chemotherapeutic drug for cancer therapy, Nanoscale, 2014, 6, 10084-10094.

100 C. Sousa, et al., Polymeric Micellar Formulation Enhances Antimicrobial and Anticancer Properties of Salinomycin, Pharm. Res., 2019, 36(6), 83.

101 S. Rezvantalab, et al., PLGA-based nanoparticles in cancer treatment, Front. Pharmacol., 2018, 9, 1-19.

102 Z. Daman, H. Faghihi and H. Montazeri, Salinomycin nanoparticles interfere with tumor cell growth and the tumor microenvironment in an orthotopic model of pancreatic cancer, Drug Dev. Ind. Pharm., 2018, 44, 1434-1442.

103 S. M. Sureban, et al., Nanoparticle-based delivery of siDCAMKL-1 increases microRNA-144 and inhibits colorectal cancer tumor growth via a Notch-1 dependent mechanism, J. Nanobiotechnol., 2011, 9, 1-13.

104 B. Wang, et al., Delivery of small interfering RNA against Nogo-B receptor via tumor-acidity responsive nanoparticles for tumor vessel normalization and metastasis suppression, Biomaterials, 2018, 175, 110-122.

105 R. K. Verma, W. Yu, A. Shrivastava, S. Shankar and R. K. Srivastava, $\alpha$-Mangostin-encapsulated PLGA nanoparticles inhibit pancreatic carcinogenesis by targeting cancer stem cells in human, and transgenic (KrasG12D, and KrasG12D/tp53R270H) mice, Sci. Rep., 2016, 6, 1-13.

106 R. K. Verma, W. Yu, S. P. Singh, S. Shankar and R. K. Srivastava, Anthothecol-encapsulated PLGA nanoparticles inhibit pancreatic cancer stem cell growth by modulating sonic hedgehog pathway, Nanomedicine, 2015, 11, 2061-2070.

107 S. Das, P. Mukherjee, R. Chatterjee, Z. Jamal and U. Chatterji, Enhancing chemosensitivity of breast cancer stem cells by downregulating SOX2 and ABCG2 using wedelolactone-encapsulated nanoparticles, Mol. Cancer Ther., 2019, 18, 680-692.

108 J. Fan, et al., Biomaterials Targeting epithelial-mesenchymal transition: Metal organic network nano-complexes for preventing tumor metastasis, Biomaterials, 2017, 139, 116-126.

109 Manuscript, A. Nanoscale, 2018, DOI: 10.1039/C8NR02708K.

110 Y. Huang, et al., ZnAs@SiO ${ }_{2}$ nanoparticles as a potential anti-tumor drug for targeting stemness and epithelialmesenchymal transition in hepatocellular carcinoma via SHP-1/JAK2/STAT3 signaling, Theranostics, 2019, 9(15), 4391-4408.

111 Y. Lu, et al., EVI1 promotes epithelial-to-mesenchymal transition, cancer stem cell features and chemo -/radioresistance in nasopharyngeal carcinoma, J. Exp. Clin. Cancer Res., 2019, 3, 1-17.

112 S. A. Shahin, et al., HHS Public Access., 2019, 14, 1381-1394. 113 C. M. Roberts, et al., Nanoparticle delivery of siRNA against TWIST to reduce drug resistance and tumor growth in ovarian cancer models, Nanomedicine, 2016, 13(3), 965-976.

114 J. Finlay, et al., HHS Public Access., 2016, 11, 1657-1666.

115 J. Finlay, et al., RNA-Based TWIST1 Inhibition via Dendrimer Complex to Reduce Breast Cancer Cell Metastasis, Biomed. Res. Int., 2015, 2015, 382745.

116 J. G. Parvani, M. D. Gujrati, M. A. Mack, W. P. Schiemann and Z. Lu, HHS Public Access., 2016, 75, 2316-2325. 
117 Z. Lu, HHS Public Access., 2020, 30, 907-919.

118 C. Ma, et al., Biomaterials Science photodynamic therapy by inhibiting epithelial, Biomater. Sci., 2017, 5, 600.

119 Y. Ma, et al., STAT3 Decoy Oligodeoxynucleotides-Loaded Solid Lipid Nanoparticles Induce Cell Death and Inhibit Invasion in Ovarian Cancer Cells, PLos One, 2015, 1-15, DOI: 10.1371/journal.pone.0124924.

120 D. Suresh, A. Zambre and S. Mukherjee, Silencing AXL by covalent siRNA-gelatin-antibody nanoconjugate inactivates mTOR/EMT pathway and stimulates p53 for TKI sensitization in NSCLC, Nanomedicine, 2019, 20, 102007.

121 Y. Li, et al., Co-delivery of microRNA-21 antisense oligonucleotides and gemcitabine using nanomedicine for pancreatic cancer therapy, Cancer Sci., 2017, 108(7), 1493-1503.

122 J. Nanobiotechnol, et al., Improving the anticancer effect of afatinib and microRNA by using lipid polymeric nanoparticles conjugated with dual $\mathrm{pH}$-responsive and targeting peptides, J. Nanobiotechnol., 2019, 1-20, DOI: 10.1186/ s12951-019-0519-6.

123 Q. Liu, et al., Biomaterials Targeted delivery of miR-200c/ DOC to inhibit cancer stem cells and cancer cells by the gelatinases-stimuli nanoparticles, Biomaterials, 2013, 34, 7191-7203.

124 S. Tang, et al., Biomaterials Inhibition of metastasis and growth of breast cancer by $\mathrm{pH}$-sensitive poly(b-amino ester) nanoparticles co-delivering two siRNA and paclitaxel, Biomaterials, 2015, 48, 1-15.

$125 \mathrm{~J}$. Shen, et al., Biomaterials Simultaneous inhibition of metastasis and growth of breast cancer by co-delivery of twist shRNA and paclitaxel using pluronic P85-PEI/TPGS complex nanoparticles, Biomaterials, 2013, 34, 1581-1590.

126 M. Alavi, N. Karimi and M. Safaei, Application of various types of liposomes in drug delivery systems, Adv. Pharm. Bull., 2017, 7, 3-9.

127 M. Kullberg, et al., Liposome Delivery of Natural STAT3 Inhibitors for the Treatment of Cancer, Pharm. Front., 2019, 3, 1-27.

$128 \mathrm{X}$. Yao, et al., GGP modified daunorubicin plus dioscin liposomes inhibit breast cancer by suppressing epithelial-mesenchymal transition, Drug Dev. Ind. Pharm., 2020, 9045, 1-44.

129 M. Song, et al., Preparation and Evaluation of Liposomes Co-Loaded with Doxorubicin, Phospholipase D Inhibitor 5Fluoro-2-Indolyl Deschlorohalopemide (FIPI) and D-Alpha Tocopheryl Acid Succinate $(\alpha$-TOS) for Anti-Metastasis, Nanoscale Res. Lett., 2019, 14, 1-13.

130 C. Y. Chang, et al., PEGylated liposome-encapsulated rhenium-188 radiopharmaceutical inhibits proliferation and epithelial-mesenchymal transition of human head and neck cancer cells in vivo with repeated therapy, Cell Death Discovery, 2018, 4(1), 100.

131 Y. A. Shen, et al., Intraperitoneal 188Re-Liposome delivery switches ovarian cancer metabolism from glycolysis to oxidative phosphorylation and effectively controls ovarian tumour growth in mice, Radiother. Oncol., 2016, 119, 282-290.

132 Z. Guo, et al., Improvement of chemosensitivity and inhibition of migration via targeting tumor epithelial-to- mesenchymal transition cells by ADH-1-modified liposomes, Drug Delivery, 2018, 25, 112-121.

133 N. G. Zheng, S. J. Mo, J. P. Li and J. L. Wu, Anti-CSC effects in human esophageal squamous cell carcinomas and Eca109/9706 cells induced by nanoliposomal quercetin alone or combined with CD 133 antiserum, Asian Pacific J. Cancer Prev., 2014, 15, 8679-8684.

134 H. Jin, et al., Targeting lipid metabolism to overcome EMTassociated drug resistance via integrin $\beta 3 /$ FAK pathway and tumor-associated macrophage repolarization using legumain-activatable delivery, Theranostics, 2019, 9, 265-278.

135 M. S. Kim, et al., Development of exosome-encapsulated paclitaxel to overcome MDR in cancer cells, Nanomedicine, 2016, 12, 655-664.

136 L. Alvarez-erviti, et al., Delivery of siRNA to the mouse brain by systemic injection of targeted exosomes, Nat. Biotechnol., 2011, 29(4), 341-345.

137 A. Srivastava, et al., Nanosomes carrying doxorubicin exhibit potent anticancer activity against human lung cancer cells, Nat. Publ. Gr., 2016, 1-15, DOI: 10.1038/ srep38541.

138 X. Wang, et al., Biomedicine \& Pharmacotherapy Prostate carcinoma cell-derived exosomal MicroRNA-26a modulates the metastasis and tumor growth of prostate carcinoma, Biomed. Pharmacother., 2019, 117, 109109.

139 C. Xie, L. Yang, D. Fengyuan, G. Xiaoshuang and L. Bo, Exosomes derived from microRNA-101-3p-overexpressing human bone marrow mesenchymal stem cells suppress oral cancer cell proliferation, invasion, and migration, Mol. Cell. Biochem., 2019, 458, 11-26.

140 F. E. I. Guo, et al., miR-101 suppresses the epithelial-tomesenchymal transition by targeting ZEB1 and ZEB2 in ovarian carcinoma, Oncol. Rep., 2014, 2021-2028, DOI: 10.3892/or.2014.3106.

141 B. L. Li, W. Lu and G. Q. Du, Loss of exosomal miR-148b from cancer-associated fibroblasts promotes endometrial cancer cell invasion and cancer metastasis, J. Cell Physiol., 2018, 1-11, DOI: 10.1002/jcp.27111.

142 W. Zhang and J. Xu, DNA methyltransferases and their roles in tumorigenesis, Biomark. Res., 2017, 1-8, DOI: 10.1186/s40364-017-0081-z.

143 Y. Ota, K. Takahashi, S. Otake, Y. Tamaki and M. Okada, Extracellular vesicle-encapsulated miR-30e suppresses cholan giocarcinoma cell invasion and migration via inhibiting epithelial-mesenchymal transition, Oncotarget., 2018, 9, 16400-16417.

144 P. Tan, et al., TRIM59 promotes breast cancer motility by suppressing p62-selective autophagic degradation of PDCD10, PLoS Bio., 2018, 16(11), e3000051.

145 L. Yuan, Y. Liu, Y. Qu, L. Liu and H. Li, Exosomes Derived From Human Umbilical Cord Mesenchymal Stem Cells Restrain Breast Cancer Progression, Front. Oncol., 2019, 9, 1-14.

146 E. J. Bunggulawa, et al., Recent advancements in the use of exosomes as drug delivery systems 06 Biological Sciences 0601 Biochemistry and Cell Biology, J. Nanobiotechnol., 2018, 16, 1-13. 